\title{
PENCILS ON AN ALGEBRAIC VARIETY AND A NEW PROOF OF A THEOREM OF BERTINI
}

\author{
BY \\ OSCAR ZARISKI( $\left.{ }^{1}\right)$
}

Introduction. A well known theorem of Bertini-Enriques on reducible linear systems of $V_{r-1}$ 's on an algebraic $V_{r}$ (i.e., linear systems in which each element is a reducible $V_{r-1}$ ) states that any such system, if free from fixed components, is composite with a pencil. The usual geometric proof of this theorem is based on another theorem of Bertini, to the effect that the general $V_{r-1}$ of a linear system cannot have multiple points outside the singular locus of the variety $V_{r}$ and the base locus of the system. This geometric proof has been subsequently completed and presented by van der Waerden under an algebraic form [3].

In this paper we give a new proof of the theorem of Bertini on reducible linear systems and we also extend this theorem to irrational pencils, i.e., pencils of genus $p>0$. Our proof does not make use of the second theorem of Bertini just quoted. In the case of pencils (linear or irrational), we first observe that a pencil $\{W\}$ on $V_{r}$ is defined by a field $P$ of algebraic functions of one variable which is a subfield of the field $\Sigma$ of rational functions on $V_{r}$. The whole proof is then essentially based on the simple remark that the pencil $\{W\}$ is composite with another pencil $\{\bar{W}\}$, defined by a field $\bar{P}$, if and only if $P$ is a subfield of $\bar{P}$. This property is a straightforward consequence of the geometric definition of composite pencils. As a matter of fact we prefer to define composite pencils by this property. At any rate, it is then true that $a$ pencil $\{W\}$ is non-composite if and only if the corresponding field $P$ is maximally algebraic in $\Sigma$. In the light of this approach to the question, the theorem of Bertini on reducible pencils is almost a direct consequence of the well known fact that an irreducible algebraic variety $V$, over a ground field $K$, is absolutely irreducible if $K$ is maximally algebraic in the field of rational functions on $V$.

In the case of linear systems of dimension $>1$ the proof is even simpler, provided use is made of a certain lemma (Lemma 5). This lemma is, however, of in terest in itself.

A sizable portion of the paper (Part I) is devoted to the development of the concept of a pencil and of the basic properties of pencils in the abstract case of an arbitrary ground field (of characteristic zero).

Presented to the Society, January 1, 1941; received by the editors July 1, 1940.

(1) Guggenheim Fellow. 


\section{Pencils of $V_{r-1}$ 's on A $V_{r}$}

1. Divisors of the first kind. Let $V$ be an irreducible algebraic $r$-dimensional variety in a projective $n$-space, over an arbitrary ground field $\mathrm{K}$ of characteristic zero. We shall assume that $V$ is normal in the projective space $\left.{ }^{2}\right)$. Let $\Sigma$ be the field of rational functions on $V$. Since $V$ is normal, any irreducible $(r-1)$-dimensional subvariety $\Gamma$ of $V$ defines a prime divisor $\mathfrak{B}$ of $\Sigma$, i.e., an homomorphic mapping of $\Sigma$ upon $\left(\Sigma_{1}, \infty\right)$, where $\Sigma_{1}$ is the field of rational functions on $\Gamma$. There is also an associated $(r-1)$-dimensional valuation $B$ of $\Sigma / \mathrm{K}$, whose valuation ring $\mathfrak{l}$ is the quotient ring $Q(\Gamma)$ of $\Gamma\left(^{3}\right)$. It is well known that $B$ (being of dimension $r-1$ ) is a discrete valuation of rank 1, i.e., its value group is the group of integers. If $\eta$ is an element of $\Sigma$ and if its value $v_{B}(\eta)$ in the valuation $B$ is $\alpha$ ( $\alpha$ a positive, negative, or zero integer), we shall say that $\eta$ has order $\alpha$ at the prime divisor $\mathfrak{B}$, or along the variety $\Gamma$. We shall also say that $\eta$ vanishes to order $\alpha$ at $\mathfrak{P}$, or along $\Gamma$, if $\alpha>0$, and that $\eta$ is infinite to the order $-\alpha$ at $\mathfrak{B}$, or along $\Gamma$, if $\alpha<0$.

The prime divisors $\mathfrak{B}$ defined as above, by means of irreducible subvarieties of $V$ of dimension $r-1$, shall be referred to as divisors of the first kind (with respect to $V\left({ }^{4}\right)$ ). Dealing with the given normal variety $V$, we shall only deal with prime divisors of the first kind with respect to $V$. Concerning these we state the following well known theorem (Krull [1, p. 137, Vollständigkeitseigenschaft]):

(2) For the definition of normal varieties see our paper [4, p. 279, 283]. Our assumption implies that $V$ is normal in the affine space, for any choice of the hyperplane at infinity. It is this weaker condition that really matters in our present treatment, rather than the condition that $V$ be normal in the projective space. The restriction to normal varieties (either in the projective or in the above affine sense) is a sound principle from the standpoint of birational geometry. We have proved, in fact, that normal varieties exist in every class of birationally equivalent varieties. We have also associated with any given variety $V$ a definite class of projectively related normal varieties, the derived normal varieties of $V[4$, p. 292], and therefore results proved for these can be readily restated as results concerning the original $V$. Finally, we point out that the class of varieties which are normal in the above affine sense includes the class of varieties which are free from singularities (in the projective space).

(3) We choose as hyperplane at infinity any hyperplane which does not contain $\Gamma$. Let $\xi_{1}, \cdots, \xi_{n}$ be the nonhomogeneous coordinates of the general point of $V$. Since the subvariety $\Gamma$ is not at infinity, it is given by a prime ideal $\mathfrak{p}$ of the ring $\mathfrak{D}=\mathbf{K}\left[\xi_{1}, \cdots, \xi_{n}\right]$. This ideal is $(r-1)-$ dimensional and is minimal in $\mathbf{D}$. Since $V$ is normal, the ring $\mathbf{D}$ is integrally closed in its quotient field $\Sigma$. Therefore the quotient ring $\mathrm{o}_{\mathfrak{p}}(=Q(\Gamma))$ is a valuation ring $\mathbb{R}$. The residue field of the corresponding valuation $B$ is the quotient field of the residue class ring $\mathfrak{o} / \mathfrak{p}$, and hence coincides with $\Sigma_{1}$. The homomorphic mapping of $\mathbb{Q}$ upon $\Sigma_{1}$ defines the prime divisor $\mathfrak{F}$.

(4) The notion of a prime divisor of the second kind with respect to $V$ is defined as follows. Any prime divisor $\mathfrak{B}$ of $\Sigma$ is by definition a homomorphic mapping of $\Sigma$ upon $\left(\Sigma_{1}, \infty\right)$, where $\Sigma_{1}$ is a field of algebraic functions of $r-1$ variables. By a proper choice of the hyperplane at infinity we may arrange matters so that none of the coordinates $\xi_{i}$ is mapped upon the symbol $\infty$. The elements of the ring $\mathfrak{D}$ (see Footnote 3 ) which are mapped upon the zero element of $\Sigma$ form then a prime ideal $\mathfrak{p}$ in $\mathfrak{D}$, and this prime ideal defines an irreducible subvariety $\Gamma$ of $V$. If $\Gamma$ is of dimension $r-1$, then our divisor $\mathfrak{B}$ is of the first kind and is uniquely determined by $\Gamma$. If $\Gamma$ is 
If an element $\eta$ of $\Sigma$ is transcendental over $\mathrm{K}$, then the set of prime divisors of the first kind along which $\eta$ is infinite is finite and non-empty.

A divisor of the first kind will be by definition a power product $\mathfrak{A}=\mathfrak{P}_{1}^{\alpha_{1}} \cdots \mathfrak{P}_{h}^{\alpha_{h}}$, where $\mathfrak{B}_{1}, \cdots, \mathfrak{P}_{h}$ are prime divisors of the first kind and where $\alpha_{1}, \cdots, \alpha_{h}$ are positive, negative, or zero integers. If all the $\alpha$ are positive integers or zero, then $\mathfrak{A}$ is said to be an integral divisor.

$\mathfrak{A}$ is said to be a principal divisor if there exists an element $\eta$ in the field $\Sigma$ such that $\eta$ has order $\alpha_{i}$ at $\mathfrak{B}_{i}(i=1,2, \cdots, h)$ and order 0 at any other prime divisor of the first kind. Notation: $\mathfrak{A}=(\eta)$.

2. Definition of a pencil. Let $\mathrm{P}$ be a subfield of $\Sigma$ containing the ground field $\mathrm{K}$ and being of degree of transcendency 1 over $\mathrm{K}$. By means of such a subfield $\mathrm{P}$ we proceed to define a collection $\{W\}$ of $(r-1)$-dimensional subvarieties $W$ of $V$, and namely one $W$ for each place, or prime divisor, of $\mathrm{P} / \mathrm{K}$.

Given an $(r-1)$-dimensional irreducible subvariety $\Gamma$ of $V$, let $\mathfrak{B}$ be the corresponding prime divisor of $\Sigma / \mathrm{K}$ and let $B$ be the valuation defined by $\mathfrak{B}$. The valuation $B$ induces a valuation $B_{1}$ in the field $P$. The valuation $B_{1}$ is either the trivial valuation, in which every element of $\mathrm{P}$ (different from 0 ) has value zero, or $B_{1}$ is a non-trivial valuation. In the first case, the mapping of $\mathbf{P}$ in the divisor $\mathfrak{P}$ is an isomorphism. In the second case, $\mathbf{P}$ is mapped by $\mathfrak{P}$ homorphically upon a field which is algebraic over $\mathrm{K}$. This mapping defines a place, or a prime divisor $\mathfrak{p}$ of $\mathrm{P} / \mathrm{K}$; it is the prime divisor which is also directly defined by the non-trivial valuation $B_{1}$. We say in this second case that the irreducible $(r-1)$-dimensional subvariety $\Gamma$, or the corresponding prime divisor $\mathfrak{B}$, corresponds to the place $\mathfrak{p}$ of $\mathrm{P} / \mathrm{K}$.

It is not difficult to see that there is at most a finite number of irreducible $V_{r-1}$ 's which correspond to a given place $\mathfrak{p}$ of $\mathrm{P} / \mathrm{K}$. In fact, let $t$ be an element of $\mathrm{P}$ whose order at the place $\boldsymbol{p}$ is positive. Then it is clear that the prime divisors of the first kind which correspond to $\mathfrak{p}$ must be among those prime factors of the principal divisor $(t)$ whose exponents are positive. The number of such prime factors is finite, since, by a previously stated theorem, $1 / t$ is infinite only at a finite number of prime divisors of the first kind.

We shall see later that to each place $\mathfrak{p}$ there corresponds at least one irreducible $V_{r-1}$ on $V$.

Let $\Gamma_{1}, \cdots, \Gamma_{m}$ be the irreducible $V_{r-1}$ 's on $V$ which correspond to the

of dimension less than $r-1$, then $\mathfrak{B}$ is of the second kind with respect to $V$. There exist infinitely many prime divisors $\mathfrak{B}$ of the second kind leading to one and the same irreducible subvariety $\Gamma$ of dimension $<r-1$.

It is well known that a prime divisor $\mathfrak{B}$ of $\Sigma / K$ defines a discrete valuation $B$ of $\Sigma$, of rank 1 . The valuation ring $\mathfrak{Q}$ of $B$ is the set of all elements of $\Sigma$ which are mapped upon elements of $\Sigma_{1}$. Our condition on the choice of the hyperplane at infinity implies that $\mathfrak{D} \subset \Omega$. The subvariety $\Gamma$ is called the center of the valuation $B$ on $V$. A prime divisor $\mathfrak{B}$ of $\Sigma / \mathrm{K}$ is of the first kind with respect to $V$ if and only if the center of the valuation defined by $\mathfrak{B}$ is a subvariety of $V$ of $d i m e n-$ sion $r-1$ (and not less). 
given place $\mathfrak{p}$ of $\mathrm{P} / \mathrm{K}$. Let $t$ be a uniformizing parameter at $\mathfrak{p}$. We attach to each $\Gamma_{i}$ a multiplicity $h_{i}$ : namely, $h_{i}$ shall be the order to which $t$ vanishes along $\Gamma_{i}$. It is clear that $h_{i}$ is a positive integer and that it is independent of the choice of the uniformizing parameter $t\left({ }^{5}\right)$. We regard the variety

$$
W_{\mathfrak{p}}=h_{1} \Gamma_{1}+\cdots+h_{m} \Gamma_{m}
$$

as the total subvariety of $V$ which corresponds to the place $p$. We define the pencil $\{W\}$ as the totality of all $W_{\mathfrak{p}}$ obtained as $\mathfrak{p}$ varies on the Riemann surface of $\mathrm{P} / \mathrm{K}$.

3. Adjunction of indeterminates. In order to derive the basic properties of pencils, we proceed to give an explicit construction of the pencil $\{W\}$ based on Kronecker's method of indeterminates. Let $\xi_{1}, \cdots, \xi_{n}$ be, as before, the nonhomogeneous coordinates of the general point of $V\left({ }^{6}\right)$. We introduce the $r-1$ forms :

$$
\eta_{i}=u_{i 1} \xi_{1}+\cdots+u_{i n} \xi_{n}, \quad i=1,2, \cdots, r-1,
$$

where the $n(r-1)$ elements $u_{i j}$ are indeterminates. These indeterminates we adjoin to the field $\Sigma$, getting a field $\Sigma^{*}=\Sigma\left(\left\{u_{i j}\right\}\right)=\Sigma\left(u_{11}, \cdots, u_{r-1, n}\right)$. The field $\Sigma^{*}$ is a pure transcendental extension of $\Sigma$, of degree of transcendency $n(r-1)$ over $\Sigma$. We also consider the fields:

$$
\begin{aligned}
& \mathrm{P}^{*}=\mathrm{P}\left(\left\{u_{i j}\right\},\left\{\eta_{i}\right\}\right), \\
& \mathrm{K}^{*}=\mathrm{K}\left(\left\{u_{i j}\right\},\left\{\eta_{i}\right\}\right) .
\end{aligned}
$$

The elements $\eta_{1}, \cdots, \eta_{r-1}$ are algebraically independent over the field $\mathrm{P}\left(\left\{u_{i j}\right\}\right)$. For, if $\eta_{1}, \cdots, \eta_{r-1}$ and the $u_{i j}$ satisfied an algebraic relation with coefficients in $\mathrm{P}$, then by specializing the $u_{i j}, u_{i j} \rightarrow c_{i j} \in \mathrm{K}$, we could get an algebraic relation over $\mathbf{P}$ between any $r-1$ of the elements $\xi_{i}\left({ }^{7}\right)$. This is impossible, since $\Sigma$ is of degree of transcendency $r-1$ over $P$.

From the algebraic independence of the $\eta_{i}$ it follows that $\Sigma^{*}$ and $\mathrm{P}^{*}$ have the same degree of transcendency over $K$, and that $P^{*}$ is of degree of transcendency 1 over $\mathrm{K}^{*}$. Hence:

(5) If $t^{\prime}$ is another uniformizing parameter at $\mathfrak{p}$, then $t^{\prime} / t$ has order zero at $\mathfrak{p}$, and consequently $t^{\prime} / t$ has also order zero along $\Gamma_{i}$.

${ }^{(6)}$ Generally speaking, the proper procedure would have been to use the homogeneous coordinates of the general point of $V$ (Zariski [4, p. 284]), thus avoiding special considerations for divisors of infinity (i.e., prime divisors of the first kind, at which at least one of the elements $\xi_{i}$ is infinite and which therefore correspond to the irreducible components of the section of $V$ with the hyperplane at infinity), However, the use of homogeneous coordinates would have required introductory definitions and proofs concerning homogeneous prime divisors and similar concepts associated with homogeneous coordinates. The size of such an introduction would be out of proportion to the limited object of this paper. We therefore prefer to deal with nonhomogeneous coordinates, also because in the present case the special considerations for the divisors at infinity are very simple and brief.

(7) See van der Waerden [2, lemma on page 17]. 
$\Sigma^{*}$ is an algebraic extension of $\mathrm{P}^{*}$. If $\mathrm{K}^{*}$ is taken as ground field, then $\mathrm{P}^{*}$ is a field of algebraic functions of one variable.

Given a prime divisor $\mathfrak{p}$ of the field $\mathrm{P} / \mathrm{K}$, we shall want to extend $\mathfrak{p}$ to a prime divisor of the field $\mathrm{P}^{*} / \mathrm{K}^{*}$. This extension is based on the following lemma, which we shall use also later on in a different connection:

Lemma 1. Let $\Omega^{*}=\Omega\left(x_{1}, \cdots, x_{m}\right)$ be a pure transcendental extension of a field $\Omega$ (i.e., $x_{1}, \cdots, x_{m}$ are algebraically independent over $\Omega$ ). Given a valuation $B$ of $\Omega$, there exists one and only one extended valuation $B^{*}$ of $\Omega^{*}$ such that the $B^{*}$-residues of $x_{1}, \cdots, x_{m}$ are $\neq \infty$ and are algebraically independent over the residue field of $B$.

Proof. Let $B^{*}$ be a valuation of $\Omega^{*}$ satisfying the conditions of the lemma, and let $\eta^{*}=f\left(x_{1}, \cdots, x_{m}\right) / g\left(x_{1}, \cdots, x_{m}\right), f, g \in \Omega\left[x_{1}, \cdots, x_{m}\right]$, be an arbitrary element of $\Omega^{*}$. Some of the coefficients of the rational function $f / g$ may have negative values in $B$. However, if we divide through $f$ and $g$ by a coefficient of minimum value, we get a rational function whose coefficients have finite $B$-residues, not all zero. We may assume then that the rational function $\mathrm{f} / \mathrm{g}$ already satisfies this condition. Let us assume that the $B$-residues of the coefficients of the denominator $g$ are all zero. Then the $B^{*}$-residue of $g\left(x_{1}, \cdots, x_{m}\right)$ is zero, since the $B^{*}$-residues of $x_{1}, \cdots, x_{m}$ are $\neq \infty$. On the other hand, the $B$-residues of the coefficients of the numerator $f$ are necessarily not all zero. Hence the $B^{*}$-residue of $f$ is different from zero, since the $B^{*}$-residues of $x_{1}, \cdots, x_{m}$ are algebraically independent over the residue field of $B$. Consequently the $B^{*}$-residue of $\eta^{*}$ is $\infty$.

On the other hand, assuming that the $B$-residues of the coefficients of $g$ are not all zero, we conclude in a similar fashion that the $B^{*}$-residue of $g$ is $\neq 0$, while the $B^{*}$-residue of $f$ is $\neq \infty$. Hence the $B^{*}$-residue of $\eta^{*}$ is $\neq \infty$.

Hence the valuation ring $\Omega^{*}$ of $B^{*}$ consists of all quotients $\mathrm{f} / \mathrm{g}$ such that the $B$-residues of the coefficients are all finite and the B-residues of the coefficients of $g$ are not all zero. This shows that $B^{*}$ is uniquely determined. On the other hand, the set of all such quotients is a ring $\Omega^{*}$ satisfying the condition that if $\eta^{*} \subsetneq \Omega^{*}$, then $1 / \eta^{*} \subset \Omega^{*}$. Hence $\Omega^{*}$ is a valuation ring. It is then immediately seen that the corresponding valuation $B^{*}$ of $\Omega^{*}$ is an extension of $B$ (i.e., $\mathbb{R}^{*} \cap \Omega$ is the valuation ring of $B$ ) and satisfies the condition of the lemma.

We point out that the value group of $B^{*}$ is the same as the value group of $B$ and that the residue field of $B^{*}$ is a pure transcendental extension of the residue field of $B$, the adjoined transcendentals being the $B^{*}$-residues of $x_{1}, \cdots, x_{m}$. The proofs of these assertions are straightforward. We also wish to point out explicitly that $B^{*}$ depends on the particular set of generators $x_{1}, \cdots, x_{m}$ of $\Omega^{*} / \Omega$. Thus, the set of generators $c x_{1}, x_{2}, \cdots, x_{m}, c \subset \Omega$, defines an extended valuation of $\Omega^{*}$ which is different from $B^{*}$, whenever $v_{B}(c) \neq 0$.

We apply the above lemma to the fields $\mathrm{P}, \mathrm{P}^{*}$, of which the second is a 
pure transcendental extension of $\mathrm{P}$. As generators of $\mathrm{P}^{*} / \mathrm{P}$ we take the elements $u_{i j}$ and $\eta_{i}$. Let $\mathfrak{p}$ be a prime divisor of $\mathrm{P} / \mathrm{K}, B_{1}$ the corresponding valuation of $\mathrm{P}, B_{1}^{*}$ the extended valuation of $\mathrm{P}^{*}$. If $\Delta_{1}$ is the residue field of $B_{1}$, then $\Delta_{1}^{*}=\Delta_{1}\left(\left\{u_{i j}^{*}\right\},\left\{\eta_{i}^{*}\right\}\right)$ is the residue field of $B_{1}^{*}$, where the $u_{i j}^{*}$ and $\eta_{i}^{*}$ are the $B_{1}^{*}$-residues of the $u_{i j}$ and $\eta_{i}$. The field $\Delta_{1}$ contains a subfield simply isomorphic to $\mathrm{K}$, which we may identify with $\mathrm{K}$. The fields $\mathrm{K}\left(\left\{u_{i j}^{*}\right\},\left\{\eta_{i}^{*}\right\}\right)$, $\mathrm{K}\left(\left\{u_{i j}\right\},\left\{\eta_{i}\right\}\right)$ are simply isomorphic, in view of the algebraic independence of the residues $u_{i j}^{*}, \eta_{i}^{*}$ with respect to $\Delta_{1}$. Hence the valuation $B_{1}^{*}$ defines a prime divisor $\mathrm{p}^{*}$ of the field $\mathrm{P}^{*} / \mathrm{K}^{*}$, i.e.; if $\mathrm{K}^{*}$ is taken as ground field and if $\mathrm{P}^{*}$ is regarded as a field of functions of one variable. Hence we have associated with each prime divisor $\mathfrak{p}$ of $\mathrm{P} / \mathrm{K}$ a prime divisor $\mathfrak{p}^{*}$ of $\mathrm{P}^{*} / \mathrm{K}^{*}$ :

$$
\mathfrak{p} \rightarrow \mathfrak{p}^{*} \text {. }
$$

Since $B_{1}^{*}$ is an extension of $B_{1}$, it is clear that distinct prime divisors of $\mathrm{P} / \mathrm{K}$ extend to distinct prime divisors of $\mathrm{P}^{*} / \mathrm{K}^{*}$.

We now consider the field $\Sigma^{*}$. It is an algebraic extension of $\mathrm{P}^{*}$. Hence the prime divisor $\mathfrak{p}^{*}$ factors into a power product of prime divisors of $\Sigma^{*} / \mathrm{K}^{*}$. Let

$$
\mathfrak{p}^{*}=\mathfrak{P}_{1}^{* h_{1}} \ldots \mathfrak{P}_{m}^{* h_{m}} \text {. }
$$

The following sections are devoted to the proof of the following assertions:

(A) Each prime divisor $\mathfrak{P}_{i}^{*}(i=1,2, \cdots, m)$ induces in $\Sigma$ a prime divisor $\mathfrak{B}_{i}$ of the first kind.

(B) The prime divisors $\mathfrak{B}_{1}, \cdots, \mathfrak{P}_{m}$ are distinct.

(C) If $\Gamma_{i}$ is the irreducible $V_{r-1}$ defined on $V$ by the prime divisor $\mathfrak{B}_{i}$, then $h_{1} \Gamma_{1}+\cdots+h_{m} \Gamma_{m}$ is the member $W_{\mathfrak{p}}$ of the pencil $\{W\}$ which corresponds to the place $\mathfrak{p}$ of $\mathrm{P} / \mathrm{K}$ (in the sense of the definition given in $\$ 2$ ).

4. The induced prime divisors $\mathfrak{B}_{i}$. We consider one of the prime divisors $\mathfrak{B}_{i}^{*}$ in $(2)$, say $\mathfrak{B}_{1}^{*}$, and we denote by $B^{*}$ the valuation of $\Sigma^{*}$ defined by $\mathfrak{B}_{1}^{*}$. In $\Sigma$, a subfield of $\Sigma^{*}, B^{*}$ induces a valuation $B$. Since $\Sigma^{*}$ is of degree of transcendency $(r-1) n$ over $\Sigma$, the residue field of $B^{*}$ can be at most of degree of transcendency $(r-1) n$ over the residue field of $B\left({ }^{8}\right)$. Since the residue field of $B^{*}$ is an algebraic extension of $\mathrm{K}^{*}$, it is of degree of transcendency $(r-1) n+(r-1)$

$\left.{ }^{8}\right)$ The proof of this assertion is immediate. Let, quite generally, $\Sigma^{*}$ be an extension field of a field $\Sigma$, of degree of transcendency $\rho$ over $\Sigma$, and let $B$ and $B^{*}$ be respectively a valuation of $\Sigma$ and an extended valuation of $\Sigma^{*}$. Let us assume that the residue field of $B^{*}$ is of degree of transcendency $\geqq \rho$ over the residue field of $B$. We consider $\rho$ elements $\omega_{1}, \cdots, \omega_{\rho}$ of $\Sigma^{*}$ whose $B^{*}$-residues $\omega_{1}^{*}, \cdots, \omega_{\rho}^{*}$ are algebraically independent over the residue field $\Delta$ of $B$. It is clear that $\omega_{1}, \cdots, \omega_{\rho}$ are algebraically independent over $\Sigma$. Hence, by the remark at the end of the proof of Lemma 1 , it follows that the residue field of the valuation induced in the field $\Sigma\left(\omega_{1}, \cdots, \omega_{\rho}\right)$ by the valuation $B^{*}$, is $\Delta\left(\omega_{1}{ }^{*}, \cdots, \omega_{\rho}^{*}\right)$. Now $\Sigma^{*}$ is an algebraic extension of the field $\Sigma\left(\omega_{1}, \cdots, \omega_{\rho}\right)$. Hence the residue field of $B^{*}$ is an algebraic extensions of $\Delta\left(\omega_{1}^{*}, \cdots, \omega_{\rho}^{*}\right)$, and therefore its degree of transcendency over $\Delta$ is exactly $\rho$. 
over $\mathrm{K}$. Hence the degree of transcendency of the residue field of $B$ over $\mathrm{K}$ must be not less than $r-1$. On the other hand, $\mathfrak{B}_{1}^{*}$ induces in $\mathrm{P}$ the non-trivial prime divisor $\mathfrak{p}$. Hence $B$, as a non-trivial valuation, is of dimension $r-1$. It defines in $\Sigma$ a prime divisor $\mathfrak{B}_{1}$. Thus the prime divisors $\mathfrak{B}_{1}^{*}, \cdots, \mathfrak{P}_{m}^{*}$ induce in $\Sigma / \mathrm{K}$ prime divisors $\mathfrak{B}_{1}, \cdots, \mathfrak{B}_{m}$.

We next show that the prime divisors $\mathfrak{B}_{1}, \cdots, \mathfrak{B}_{m}$ are of the first kind with respect to $V$. Let us consider for instance the divisor $\mathfrak{B}_{1}$. We examine separately two cases, according as the center of $\mathfrak{P}_{1}$ (i.e., of the corresponding valuation [see Footnote 4]) on $V$ is or is not at finite distance.

First case. The center of $\mathfrak{B}_{1}$ is at finite distance (i.e., it does not lie in the hyperplane at infinity). In this case the $B$-residues $\xi_{1}^{*}, \cdots, \xi_{n}^{*}$ of $\xi_{1}, \cdots, \xi_{n}$ are all different from $\infty$. Let $u_{j}^{*}, \eta_{i}^{*}$ be the $B^{*}$-residues of the elements $u_{i j}, \eta_{i}$. These $B^{*}$-residues are algebraically independent over $\mathrm{K}$. On the other hand we have: $\eta_{i}^{*}=u_{i 1}^{*} \xi_{1}^{*}+\cdots+u_{i n}^{*} \xi_{n}^{*}$. Hence $r-1$ of the elements $\xi_{i}^{*}$ must be algebraically independent over $\mathrm{K}$. This shows that the center of $\mathfrak{B}_{1}$ on $V$ is of dimension $r-1$, whence $\mathfrak{P}_{1}$ is of the first kind.

Second case. The center of $\mathfrak{B}_{1}$ is at infinity. In this case some of the elements $\xi_{i}$ have negative values in $B$. Without loss of generality we may assume that $0>v_{B}\left(\xi_{1}\right)=\min \left(v_{B}\left(\xi_{1}\right), \cdots, v_{B}\left(\xi_{n}\right)\right)$. We consider the following projective transformation of coordinates:

$$
\xi_{1}^{\prime}=1 / \xi_{1}, \quad \xi_{i}^{\prime}=\xi_{i} / \xi_{1}, \quad i=2, \cdots, n .
$$

With respect to the new nonhomogeneous coordinates $\xi_{i}^{\prime}$ the center of $\mathfrak{P}_{1}$ is at finite distance. To show that $\mathfrak{P}_{1}$ is of the first kind, we have to show that among the $B$-residues $\xi_{1}^{\prime *}, \xi_{2}^{\prime *}, \cdots, \xi_{n}{ }^{*}$ of the $\xi_{i}^{\prime}$ there are $r-1$ which are algebraically independent over $\mathrm{K}$. To show this we observe that we have:

$$
\eta_{i}=\frac{u_{i 1}+u_{i 2} \xi_{2}^{\prime}+\cdots+u_{i n} \xi_{n}^{\prime}}{\xi_{1}^{\prime}} .
$$

Hence passing to the $B^{*}$-residues and noting that the $B$-residue of $\xi_{1}^{\prime}$ is zero we find:

$$
u_{i 1}^{*}+u_{i 2}^{*} \xi_{2}^{\prime *}+\cdots+u_{i n}^{*} \xi_{n}^{\prime *}=0 .
$$

These relations show that the $r-1$ elements $u_{i 1}^{*}$ belong to the field $\mathrm{K}\left(\left\{u_{i j}^{*}\right\}\right.$, $\left.j>1 ; \xi_{2}^{\prime *}, \cdots, \xi_{n}^{\prime *}\right)$. Since the $(r-1) n$ elements $u_{i j}^{*}$ are algebraically independent over $\mathrm{K}, r-1$ of the residues $\xi_{2}^{\prime}{ }^{*}, \cdots, \xi_{n}{ }^{*}$ must be algebraically independent over $K$, q.e.d.

Thus assertion (A) is fully established.

5. Proof of (B). Let us consider one of the divisors $\mathfrak{B}_{1}, \cdots, \mathfrak{B}_{m}$, say $\mathfrak{B}_{1}$, and let, as before, $B$ be the corresponding valuation of $\Sigma$, while $B^{*}$ is the valuation of $\Sigma^{*}$ defined by $\mathfrak{B}_{1}^{*}$. We know that $B^{*}$ is an extended valuation of $B$. We also know that $\Sigma^{*}$ is a pure transcendental extension of $\Sigma$. By Lemma 1 , 
$B^{*}$ is uniquely determined by $B$ if a set of generators of $\Sigma^{*}$ over $\Sigma$ is known whose $B^{*}$-residues are algebraically independent over the residue field of $B$. Since $\mathfrak{B}_{1}^{*}, \cdots, \mathfrak{P}_{m}^{*}$ are distinct divisors, the assertion (B) will be proved if we show that it is possible to exhibit such a set of generators which depend only on $B$. Let us first suppose that the center of $B$ is at finite distance. The residue field of $B$ is the field $\mathrm{K}\left(\xi_{1}^{*}, \ldots, \xi_{n}^{*}\right)$, where the $\xi_{i}{ }^{*}$ are the $B$-residues of the $\xi_{i}$. Since the field $\mathrm{K}\left(\left\{u_{i j}^{*}\right\},\left\{\eta_{i}^{*}\right\}\right)$ is of degree of transcendency $(r-1) n+(r-1)$ over $\mathrm{K}$ and since this field is contained in the field $\mathrm{K}\left(\left\{u_{i j}^{*}\right\}, \xi_{1}^{*}, \cdots, \xi_{n}^{*}\right)$, it follows that the $(r-1) n$ residues $u_{i j}^{*}$ are algebraically independent over the field $\mathrm{K}\left(\xi_{1}^{*}, \cdots, \xi_{n}^{*}\right)$ (since this last field is of degree of transcendency $r-1$ over $\mathrm{K})$. Hence, if the center of $B$ is at finite distance, the $(r-1) n$ elements $u_{i j}$ form a set of generators of the desired nature.

Let us now assume that the center of $B$ is at infinity, and let, say, $0>v_{B}\left(\xi_{1}\right)=\min \left(v_{B}\left(\xi_{1}\right), \cdots, v_{B}\left(\xi_{n}\right)\right)$. In this case the $B^{*}$-residues $u_{i j}^{*}$ of the $u_{i j}$ are not algebraically independent over the residue field $\mathrm{K}\left(\xi_{2}{ }^{*}, \cdots, \xi_{n}{ }^{*}\right)$ of $B$, in view of (5). However, the elements $u_{i j}, j>1$, and $\eta_{1}, \cdots, \eta_{r-1}$ also form a set of generators of $\Sigma^{*}$ over $\Sigma$. We assert that the $B^{*}$-residues of these elements are algebraically independent over $\mathrm{K}\left(\xi_{2}^{\prime}{ }^{*}, \ldots, \xi_{n}{ }^{*}\right)$. To show this, we observe that in view of the relations (5), the field

$$
\Delta^{*}=\mathrm{K}\left(\xi_{2}^{\prime *}, \cdots, \xi_{n}^{\prime *} ;\left\{u_{i j}^{*}\right\}, j>1 ; \eta_{1}^{*}, \cdots, \eta_{r-1}^{*}\right)
$$

contains the subfield $\mathrm{K}\left(\left\{u_{i j}^{*}\right\}, \eta_{1}^{*}, \cdots, \eta_{r-1}^{*}\right)$. Since this last field is of degree of transcendency $(r-1) n+(r-1)$ over $\mathrm{K}$, and since $\mathrm{K}\left(\xi_{2}{ }^{*}, \cdots, \xi_{n}{ }^{*}\right)$ is of degree of transcendency $r-1$ over $\mathrm{K}$, it follows that the $(n-1) r$ residues of $u_{i j}^{*}(j>1), \eta_{1}^{*}, \cdots, \eta_{r-1}^{*}$, are algebraically independent over $\mathrm{K}\left(\xi_{2}^{\prime *}, \cdots, \xi_{n}{ }^{*}\right)$, q.e.d.

The proof of assertion (B) is now complete.

6. Proof of $(\mathrm{C})$. Let $\Gamma_{1}, \cdots, \Gamma_{m}$ be the $(r-1)$-dimensional irreducible subvarieties of $V$ which correspond to the prime divisors $\mathfrak{B}_{1}, \cdots, \mathfrak{P}_{m}$. Let $W_{\mathfrak{p}}$ be the member of the pencil $\{W\}$ which corresponds to the place $\mathfrak{p}$ of $\mathrm{P} / \mathrm{K}$, in the sense of the definition given in $\$ 2$. Since each prime divisor $\mathfrak{B}_{i}{ }^{*}$, and hence also each $\mathfrak{B}_{i}$, induces in $\mathrm{P} / \mathrm{K}$ the prime divisor $\mathfrak{p}$, it follows that $\Gamma_{1}, \cdots, \Gamma_{m}$ are components of $W_{\mathfrak{p}}$.

Let $t$ be an element of $\mathrm{P}$ which has order 1 at $\mathfrak{p}$. Then $t$, considered as an element of $\mathrm{P}^{*}$, also has order 1 at $\mathfrak{p}^{*}$. In view of (2), $t$ has order $h_{i}$ at $\mathfrak{B}_{i}{ }^{*}$. Since the value group of the valuation of $\Sigma$ defined by $\mathfrak{P}_{i}$ is the same as the value group of the valuation of $\Sigma^{*}$ defined by $\mathfrak{B}_{i}^{*}$ (see remark at the end of the proof of Lemma 1), it follows that $t$ has also order $h_{i}$ at $\mathfrak{B}_{i}$. We conclude that $\Gamma_{i}$ is an $h_{i}$-fold component of $W_{\mathfrak{p}}$ (in the sense of the definition of $\$ 2$ ).

To complete the proof of (C), we have to show that if $\Gamma$ is an irreducible component of $W_{\mathfrak{p}}$, then $\Gamma$ coincides with one of the varieties $\Gamma_{1}, \cdots, \Gamma_{m}$. Let $\mathfrak{B}$ be the prime divisor of $\Sigma$ defined by $\Gamma$ and let $B$ be the corresponding valua- 
tion of $\Sigma$. Let $B^{*}$ be the extended valuation of $B$ in $\Sigma^{*}$ defined by the following condition: (1) if $\Gamma$ is at finite distance, then the $B^{*}$-residues of the $(r-1) n$ elements $u_{i j}$ are algebraically independent over the residue field of $B$; (2) if $\mathfrak{B}$ is a divisor at infinity, and if, say, $0>v_{B}\left(\xi_{1}\right)=\min \left(v_{B}\left(\xi_{1}\right), \cdots, v_{B}\left(\xi_{n}\right)\right)$, then the $B^{*}$-residues of the $(r-1) n$ elements $u_{i j}(j>1), \eta_{1}, \cdots, \eta_{r-1}$, are algebraically independent over the residue field of $B$.

In view of Lemma 1 , the valuation $B^{*}$ is well defined in either case. We assert that the $B^{*}$-residues of the elements $u_{i j}, \eta_{i}$ are algebraically independent over $\mathrm{K}$.

Proof. First case. $\mathfrak{P}$ is a divisor at finite distance. Let $u_{i j}^{*}, \eta_{i}^{*}, \xi_{j}^{*}$ denote $B^{*}$-residues of the corresponding elements $u_{i j}, \eta_{i}, \xi_{j}$. We have $\eta_{i}^{*}=u_{i 1}^{*} \xi_{1}^{*}+\cdots+u_{i n}^{*} \xi_{n}^{*}$. By our construction of $B^{*}$, the elements $u_{i j}^{*}$ are algebraically independent over the field $\mathrm{K}\left(\xi_{1}^{*}, \cdots, \xi_{n}^{*}\right)$. The rest of the proof is based on the van der Waerden lemma quoted in Footnote 7 and is identical with the proof that $\eta_{1}, \cdots, \eta_{r-1}$ are algebraically independent over $\mathrm{P}\left(\left\{u_{i j}\right\}\right)$.

Second case. $\mathfrak{B}$ is a divisor at infinity, and $0>v_{B}\left(\xi_{1}\right)=\min \left(v_{B}\left(\xi_{1}\right), \cdots, v_{B}\left(\xi_{n}\right)\right)$. By our construction of $B^{*}$, the residues $u_{i j}^{*}, j>1$, are algebraically independent over the field $\mathrm{K}\left(\xi_{2}^{\prime *}, \cdots, \xi_{n}^{\prime *}\right)$, where the $\xi_{i}^{\prime}$ are given by the relations $\left.(3){ }^{9}\right)$. By a specialization argument similar to the one used in the preceding case and based on van der Waerden's lemma, it follows that the $r-1$ forms $u_{i 2}^{*} \xi_{2}^{\prime *}+\cdots+u_{\imath n}^{*} \xi_{n}{ }^{*}$ are algebraically independent over the field $\mathrm{K}\left(\left\{u_{i j}^{*}\right\}\right.$, $j>1)$. But these forms are equal to the elements $-u_{i 1}^{*}$, in view of the relations (5). Hence the $(r-1) n$ elements $u^{*}$ are algebraically independent over $\mathrm{K}$. On the other hand, the residues $\eta_{1}^{*}, \cdots, \eta_{r-1}^{*}$ are, by hypothesis, algebraically independent over the field $\mathrm{K}\left(\xi_{2}{ }^{*}, \cdots, \xi_{n}{ }^{*} ;\left\{u^{*}\right\}, j>1\right)$. Hence $a$ fortior $i$ these residues are algebraically independent over the subfield $\mathrm{K}\left(\left\{u^{*}\right\}\right)$, and this proves our assertion.

The fact that the $B^{*}$-residues $u_{i j}^{*}, \eta_{i}^{*}$ are algebraically independent over $\mathrm{K}$, implies that $B^{*}$ is a valuation of $\Sigma^{*}$ over the ground field $\mathrm{K}^{*}$, i.e., $B^{*}$ defines a prime divisor $\mathfrak{B}^{*}$ of $\Sigma^{*} / \mathrm{K}^{*}$. This prime divisor $\mathfrak{P}^{*}$ lies over a definite prime divisor ${ }^{*} \mathfrak{p}^{*}$ of $\mathrm{P}^{*} / \mathrm{K}^{*}$. To show that $\mathfrak{B}^{*}$ coincides with one of the prime divisors $\mathfrak{P}_{1}^{*}, \ldots, \mathfrak{B}_{m}^{*}$, it is only necessary to show that ${ }^{*} \mathfrak{p}^{*}=\mathfrak{p}^{*}$. Now both $\mathfrak{p}^{*}$ and ${ }^{*} \mathfrak{p}^{*}$ induce in the field $\mathrm{P} / \mathrm{K}$ one and the same prime divisor, namely $\mathfrak{p}$, since, by hypothesis, $\Gamma$ is a component of $W_{\mathfrak{p}}$. In view of Lemma 1 , the relation ${ }^{*} p^{*}=p^{*}$ will follow if we prove that the residues of the $(n+1)(r-1)$ elements $u_{i j}, \eta_{i}$ in the divisor ${ }^{*} p^{*}$ are algebraically independent over the residue field of the divisor $\mathfrak{p}$. But this is obvious, since this last residue field is algebraic over $\mathrm{K}$ and since we have just proved that the $B^{*}$-residues of the elements $u_{i j}, \eta_{i}$ are algebraically independent over $\mathrm{K}$. This completes the proof of assertion (C).

7. Order of $W_{\mathfrak{p}}$. Let $\eta_{i}=u_{i 1} \xi_{1}+\cdots+u_{i n} \xi_{n}, i=1,2, \cdots, r$, where the

(9) Note that since $V$ is normal, $\mathrm{K}\left(\xi_{1}^{\prime *}, \cdots, \xi_{n}^{\prime *}\right)$ is the residue field of $B$, and also that $\xi_{1}^{\prime *}=0$. 
$n r$ elements $u_{i j}$ are indeterminates. We define the order of $V$ as the relative degree $\left[\Sigma\left(\left\{u_{i j}\right\}\right): \mathrm{K}\left(\left\{u_{i j}\right\}, \eta_{1}, \cdots, \eta_{r}\right)\right]$. If we apply this definition to each irreducible component $\Gamma_{i}$ of $W_{\mathfrak{p}}$, we can speak of the order of $W_{\mathfrak{p}}$ : it shall be, by definition, the sum of the orders of $\Gamma_{1}, \cdots, \Gamma_{m}$, each counted to its multiplicity $h_{1}, \cdots, h_{m}$, respectively.

Given a field $\Omega$ of algebraic functions of one variable, over a ground field $\mathrm{K}$, and given a prime divisor $\mathfrak{p}$ in $\Omega / \mathrm{K}$, by the degree of $\mathfrak{p}$ we mean the relative degree $[\Delta: \mathrm{K}]$, where $\Delta$ is the residue field of $\mathfrak{p}$.

From this definition it follows immediately that the prime divisor $\mathfrak{p}$ of $\mathrm{P} / \mathrm{K}$ and the extended prime divisor $\mathrm{p}^{*}$ of $\mathrm{P}^{*} / \mathrm{K}^{*}$ have the same degree, since the residue field of $\mathfrak{p}^{*}$ is a pure transcendental extension of the residue field of $p$ (see Lemma 1 and the application to the fields $\mathrm{P}, \mathrm{P}^{*}, \S 3$ ). Let $d$ be the common degree of $\mathfrak{p}$ and $\mathfrak{p}^{*}$. Let $d_{1}, \cdots, d_{m}$ be the degrees of the prime divisors $\mathfrak{B}_{1}^{*}, \cdots, \mathfrak{P}_{m}^{*}$ respectively. It is well known that the relation (2) implies

$$
\nu d=h_{1} d_{1}+\cdots+h_{m} d_{m},
$$

where $\nu$ is the relative degree $\left[\Sigma^{*}: \mathrm{P}^{*}\right]$. The relation (6) has a geometric interpretation, namely, that the right-hand side $h_{1} d_{1}+\cdots+h_{1_{n}} d_{1 n}$ is the order of $W_{\mathfrak{p}}$. To prove this, we have to show that $d_{i}$ is the order of $\Gamma_{i}$.

Proof. First case. $\Gamma_{1}$ is at finite distance. The nonhomogeneous coordinates of the general point of $\Gamma_{1}$ are $\xi_{1}^{*}, \cdots, \xi_{n}^{*}$, where the $\xi_{i}^{*}$ are the residues of the $\xi_{i}$ in the valuation $B$ defined by the prime divisor $\mathfrak{B}_{1}$. The field $\Delta=\mathbf{K}\left(\xi_{1}^{*}, \cdots, \xi_{n}^{*}\right)$ is the residue field of $B$. The residue field of the extended valuation $B^{*}$ in $\Sigma^{*}$ is $(\S 5) \mathrm{K}\left(\xi_{1}^{*}, \cdots, \xi_{n}^{*},\left\{u_{i j}^{*}\right\}\right)$. The degree of $\mathfrak{B}_{1}^{*}$ is the relative degree

$$
d_{1}=\left[\Delta\left(\left\{u_{i j}^{*}\right\}: \mathrm{K}\left(\left\{u_{i j}^{*}\right\},\left\{\eta_{i}^{*}\right\}\right)\right] .\right.
$$

Since the $u_{i j}^{*}$ are algebraically independent over $\Delta$, the relation above shows that $d_{1}$ is also the order of $\Gamma_{1}$.

Second case. $\Gamma_{1}$ is at infinity, and $0>v_{B}\left(\xi_{1}\right)=\min \left(v_{B}\left(\xi_{1}\right), \cdots, v_{B}\left(\xi_{n}\right)\right)$. In this case the general point of $\Gamma_{1}$ is $\left(\xi_{1}^{\prime *}, \xi_{2}^{\prime *}, \cdots, \xi_{n}{ }^{*}\right)$, where the $\xi_{i}^{\prime}$ are defined by the transformation (3). But since $\xi_{1}^{\prime *}=0, \Gamma_{1}$ actually lies in an $S_{n-1}$ (in the hyperplane at infinity of $\left.S_{n}\right)$, and the general point of $\Gamma_{1}$ is $\left(\xi_{2}^{\prime *}, \cdots, \xi_{n}^{\prime *}\right)$. The field $\Delta=\mathrm{K}\left(\xi_{2}^{\prime *}, \cdots, \xi_{n}{ }^{*}\right)$ is the residue field of $B$. By $\S 5$, the residue field of $B^{*}$ is $\Delta\left(\left\{u_{i j}^{*}\right\}, j>1 ;\left\{\eta_{i}^{*}\right\}\right)$, and we have

$$
d_{1}=\left[\Delta\left(\left\{u_{i j}^{*}\right\}, j>1 ;\left\{\eta_{i}^{*}\right\}\right): \mathrm{K}\left(\left\{u_{i j}^{*}\right\},\left\{\eta_{i}^{*}\right\}\right)\right] .
$$

Since $\eta_{1}^{*}, \cdots, \eta_{r-1}^{*}$ are algebraically independent over the field $\Delta\left(\left\{u_{i j}^{*}\right\}, j>1\right)$, we can also write:

$$
d_{1}=\left[\Delta\left(\left\{u_{i j}^{*}\right\}, j>1\right): \mathrm{K}\left(\left\{u_{i j}^{*}\right\}, j>1 ;\left\{u_{i 1}^{*}\right\}\right)\right] .
$$

The assertion that $d_{1}$ is the order of $\Gamma_{1}$ now follows from the relations (5). 
In view of (6), we may now state the following result: the quotient

$$
\frac{\text { order of } W_{\mathfrak{p}}}{\text { degree of } \mathfrak{p}}
$$

is the same for all members of the pencil $\{W\}$. In the particular case when $\mathrm{K}$ is algebraically closed, the degree of $\mathfrak{p}$ is always 1 , and hence all the members of the pencil $\{W\}$ have the same order.

8. The base points of the pencil $\{W\}$. A point $A$ of $V$ is said to be a base point of the pencil $\{W\}$ if every $W_{\mathfrak{p}}$ is on $A$. Let $A$ be a point of $V$ and let $Q(A)=\Im$ be the quotient ring of $A$. We consider the intersection $R=\Im \cap \mathrm{P}$. We prove the following theorem:

$A$ is a base point of $\{W\}$ if and only if the ring $R$ is an algebraic extension of $\mathrm{K}$. If $A$ is not a base point of $\{W\}$, then there exists one and only one $W_{\mathfrak{p}}$ which passes through $A$.

Proof. Let us first assume that $R$ contains elements which are transcendental over $\mathrm{K}$, whence $R$ is of degree of transcendency 1 over $\mathrm{K}$. Since $V$ is normal, $\mathfrak{\Im}$ is in tegrally closed in $\Sigma$. Consequently

(a) $R$ is integrally closed in $\mathbf{P}$.

Since every element of $\mathbf{P}$ is algebraic over $R$, it follows by (a) that

(b) $\mathrm{P}$ is the quotient field of $R$.

Let $\omega$ be an arbitrary element of $R$. Since $\Im$ is the quotient ring of a zerodimensional ideal, there exists a polynomial $f(\omega)$, with coefficients in $K$, such that $f(\omega)$ is a non-unit in $\Im$. Hence $1 / f(\omega) \overleftarrow{\Im} \Im$, whence $1 / f(\omega)$ is an element of $P$ which is not in $R$. Therefore

(c) $R$ is a proper subring of $\mathrm{P}$.

The non-units of $\Im$ form an ideal. Hence also

(d) The non-units of $R$ form an ideal.

Since $P$ is a field of algebraic functions of one variable, the properties (a), (b), (c), (d) of $R$ imply that $R$ is a valuation ring. Let $p$ be the corresponding prime divisor of $\mathrm{P} / \mathrm{K}$.

If $W$ is a member of the pencil and if $W \neq W_{\mathfrak{p}}$, then $W$ does not pass through $A$. For, let $\Gamma$ be any irreducible component of $W$. The quotient ring $Q(\Gamma)$ is the valuation ring of the valuation defined by $\Gamma$ (see Footnote 3 ). Since $W \neq W_{\mathfrak{p}}$, the intersection $Q(\Gamma) \cap \mathrm{P}$ is different from $R$ (this intersection is the valuation ring associated with a prime divisor of $\mathrm{P} / \mathrm{K}$ different from $\mathfrak{p})$. Hence $Q(\Gamma) \cap \mathrm{P} D R$, whence $Q(\Gamma) D \Im$. Therefore $A$ is not on $\Gamma$. Since this holds true for any component $\Gamma$ of $W, A$ is not on $W$, as was asserted.

The variety $W_{\mathfrak{p}}$ passes through $A$. Let $t$ be a non-unit of $R$. Then $t$ is also a non-unit of $\Im$. Therefore at least one prime factor in the numerator of the principal divisor $(t)$ must arise from a minimal prime of $\Im$. This minimal prime corresponds to an irreducible $(r-1)$-dimensional variety $\Gamma$ passing 
through $A$. The order of $t$ along $\Gamma$ is positive, whence the valuation of $\Sigma / \mathrm{K}$ defined by $\Gamma$ induces in $\mathrm{P} / \mathrm{K}$ a non-trivial valuation. The corresponding prime divisor of $\mathrm{P} / \mathrm{K}$ must coincide with $\mathfrak{p}$, since $Q(\Gamma) \supset Q(A) \supset R$. Hence $\Gamma$ is a component of $W_{\mathfrak{p}}$, and thus $W_{\mathfrak{p}}$ is on $A$.

Let us suppose now that $R$ is an algebraic extension of $\mathrm{K}$. Let $\mathfrak{p}$ be an arbitrary prime divisor of $\mathrm{P} / \mathrm{K}$. Let $t$ be an element of $\mathrm{P}$ which has positive order at $\mathfrak{p}$ and nonpositive order at any other prime divisor of $\mathrm{P} / \mathrm{K}$. It is well known that such elements $t$ exist. The element $t$ is transcendental over $\mathrm{K}$. Hence $1 / t \nsubseteq R$, and consequently $1 / t \nsubseteq \Im$. This implies that one of the prime factors in the numerator of the principal divisor $(t)$ must arise from an irreducible subvariety $\Gamma$ (of dimension $r-1$ ) which passes through $A$. Since $t$ has positive order along $\Gamma$, it follows as before that $\Gamma$ is a component of some member of the pencil, and this member must be $W_{\mathfrak{p}}$, since $\mathfrak{p}$ is the only prime divisor of $\mathrm{P} / \mathrm{K}$ at which $t$ has positive order. Hence $W_{\mathfrak{p}}$ is on $A$. Since $\mathfrak{p}$ was an arbitrary prime divisor of $\mathrm{P} / \mathrm{K}$, we conclude that $A$ is a base point of the pencil $\{W\}$. This completes the proof of our theorem.

It is not difficult to see that the base points of the pencil $\{W\}$ form an algebraic subvariety of $V$, of dimension $\leqq r-2$. To see this, we fix an element $t$ in $\mathrm{P}$ which is transcendental over $\mathrm{K}$. Let $M_{1}, \cdots, M_{s}$ and $N_{1}, \cdots, N_{\sigma}$ be the irreducible $(r-1)$-dimensional subvarieties of $V$ along which $t$ has respectively positive or negative order, and let $H$ be the intersection of the two varieties $M_{1}+\cdots+M_{s}$ and $N_{1}+\cdots+N_{\sigma}$. Clearly, $H$ is of dimension $\leqq r-2$. We assert that $H$ is the base locus of the pencil $\{W\}$. Namely, if $A$ is a base point of $\{W\}$, then, by the preceding proof, neither $t$ nor $1 / t$ can belong to the quotient ring $Q(A)$, since both $t$ and $1 / t$ are transcendentals over K. Consequently the point $A$ must lie on at least one of the varieties $M_{i}$ and also on at least one of the varieties $N_{j}$. Therefore $A$ is on $H$. Conversely, assume that $A$ is on $H$, and let, say, $A$ be on $M_{1}$ and $N_{1}$. It is clear that $M_{1}$ belongs to some member $W_{\mathfrak{p}}$ of the pencil, such that $t$ has positive order at $\mathfrak{p}$. Similarly $N_{1}$ belongs to some member $W_{\mathfrak{p}^{\prime}}$ of the pencil such that $t$ has negative order at $\mathfrak{p}^{\prime}$. Hence the point $A$ lies on two distinct members of the pencil, $W_{\mathfrak{p}}$ and $W_{\mathfrak{p}^{\prime}}$, and consequently $A$ is a base point.

Remark. Pencil with fixed components. Let $\Gamma_{0}$ be a fixed $(r-1)$-dimensional subvariety of $V$ and let $\{W\}$ be a pencil. It is convenient to regard also the collection of varieties $W^{\prime}=W+\Gamma_{0}$ as a pencil. All members of this new pencil $\left\{W^{\prime}\right\}$ have a fixed component, namely $\Gamma_{0}$. The base locus of $\left\{W^{\prime}\right\}$ is naturally no longer of dimension $\leqq r-2$, since $\Gamma_{0}$ itself is a part of the base locus.

The pencils considered heretofore are pencils without fixed components.

\section{The theorem of Bertini for PENCILS}

9. Composite pencils. Let $\{W\},\left\{W^{*}\right\}$ be two distinct pencils on $V$, both free from fixed components, and let $\mathrm{P}, \mathrm{P}^{*}$ be the corresponding subfields of $\Sigma$, of degree of transcendency 1 over $\mathrm{K}$. 
Definition. The pencil $\{W\}$ is composite with the pencil $\left\{W^{*}\right\}$ if $\mathbf{P}$ is a subfield of $\mathbf{P}^{*}$.

The geometric significance of this definition is straightforward. If $\mathbf{P}$ is a subfield of $\mathrm{P}^{*}$, then $\mathrm{P}^{*}$ is an algebraic extension of $\mathrm{P}$, since both have degree of transcendency 1 over $\mathrm{K}$. Given a prime divisor $\mathfrak{p}$ of $\mathrm{P} / \mathrm{K}$, it factors in $\mathrm{P}^{*} / \mathrm{K}$ :

$$
\mathfrak{p}=\mathfrak{p}_{1}^{* p_{1}} \cdots \mathfrak{p}_{s}^{* p_{s}},
$$

where, if $\mathfrak{p}$ is of degree $d$ and $\mathfrak{p}_{i}^{*}$ is of degree $d_{i}^{*}$, then $\rho_{1} d_{1}^{*}+\cdots+\rho_{8} d_{s}^{*}=\nu d$, $\nu=\left[\mathrm{P}^{*}: \mathrm{P}\right]$. Consider the varieties $W_{\mathfrak{p}}, W_{p_{\mathfrak{i}}}^{*}$. If $\Gamma^{*}$ is an irreducible component of $W_{\mathfrak{p}^{*}}$, then $\Gamma^{*}$ is also a component of $W_{\mathfrak{p}}$, since $\mathfrak{p}_{i}^{*}$ lies over $\mathfrak{p}$. Moreover, if $\Gamma^{*}$ occurs in $W_{p^{*}}^{*}$ to the multiplicity $h_{i}$, it must occur in $W_{\mathfrak{p}}$ to the multiplicity $h_{i} \rho_{i}$, since if an element of $\mathrm{P}$ has order 1 at $\mathfrak{p}$, it has order $\rho_{i}$ at $\mathfrak{p}_{i}^{*}$. Finally, if $\Gamma$ is an irreducible component of $W_{\mathfrak{p}}$ and if $\mathfrak{B}$ is the corresponding prime divisor of $\Sigma$, then $\mathfrak{P}$ induces in $\mathrm{P}$ the prime divisor $\mathfrak{p}$. Therefore $\mathfrak{B}$ must induce in $\mathrm{P}^{*}$ one of the prime divisors $\mathfrak{p}_{i}^{*}$, and consequently $\Gamma$ is a component of one of the varieties $W_{p^{*}}^{*}$. We conclude that

$$
W_{\mathfrak{p}}=\rho_{1} W_{\mathfrak{p}_{\mathrm{i}}}^{*}+\cdots+\rho_{s} W_{\mathfrak{p}_{\mathrm{i}}^{*}}^{*},
$$

i.e., if the pencil $\{W\}$ is composite with the pencil $\left\{W^{*}\right\}$, then every $W$ consists of a certain number of members of the pencil $\left\{W^{*}\right\}$.

In general this number $\rho_{1}+\cdots+\rho_{s}$ depends on $W_{\mathfrak{p}}$. But if $\mathrm{K}$ is algebraically closed, then $d=d_{i}^{*}=1$, whence $\rho_{1}+\cdots+\rho_{s}=\nu$, the relative degree $\left[\mathrm{P}^{*}: \mathrm{P}\right]\left({ }^{10}\right)$. For particular prime divisors $\mathfrak{p}$ it may happen that $\rho_{1}+\cdots+\rho_{\mathrm{s}}$ $=1$, i.e., that $W_{\mathfrak{p}}$ itself is a member of the pencil $\left\{W^{*}\right\}$. This can happen even for infinitely many prime divisors $\mathfrak{p}$. But it is easily seen that there always exist infinitely many prime divisors $\mathfrak{p}$ for which $\rho_{1}+\cdots+\rho_{s}>1$, so that in a composite pencil there always exist infinitely many $W_{\mathfrak{y}}$ which are reducible over $\mathrm{K}\left({ }^{11}\right)$.

(10) There is one case in which our definition of composite jencils must be slightly modified. It is the case in which $\Sigma$ is a field of functions of one variable. Since $\Sigma$ itself is of degree of transcendency 1 , it can be taken as field $P$ and it therefore defines a pencil of zero-dimensional varieties on the curve $V$. This pencil is nothing else than the Riemann surface $R$ of $\Sigma$, and every other pencil on $V$ would be, according to our definition, composite with $R$. To avoid this undesirable conclusion, one must define composite pencils on an algebraic curve (i.e., composite involutions of sets of points) by the condition that the field P defining the pencil is not a maximal subfield of $\Sigma$.

(11) Proof. Let $x$ be a transcendental element of $\mathrm{P}$ and let $\subseteq$ and $\Im^{*}$ denote the rings of integral functions of $x$ in $\mathrm{P}$ and in $\mathrm{P}^{*}$ respectively. Let $\omega$ be a primitive element of $\mathfrak{S}^{*}$, with respect to $P$, which is not algebraic over $K$, and let $D$ be the discriminant of the base $1, \omega, \cdots, \omega^{\nu-1}$. Let $\mathfrak{p}^{*}$ be a prime $\Im^{*}$-ideal at which the residue of $\omega$ is an element of $\mathbf{K}$ and such that $D \not \equiv 0\left(\mathfrak{p}^{*}\right)$. There exist infinitely many such prime ideals $\mathfrak{p}^{*}$ since $\mathrm{K}$ contains infinitely many elements and since the numbers of prime divisors of $\mathrm{P}^{*}$ which are at infinity with respect to $\mathfrak{S}^{*}$ is finite. Let finally $\mathfrak{p}^{*} \cap \mathfrak{S}=\mathfrak{p}$. The assumption $D \not \equiv 0(\mathfrak{p})$ implies that $1, \omega, \cdots, \omega^{-1}$ 
The condition that $\{W\}$ is composite with $\left\{W^{*}\right\}$ implies, at any rate, that the field $\mathrm{P}$ is not maximally algebraic in $\Sigma$, since $\mathrm{P}^{*}$ is a proper algebraic extension of $\mathbf{P}$. Conversely, if $\mathbf{P}$ is not maximally algebraic in $\Sigma$, and if $\mathrm{P}^{*}$ denotes the relative algebraic closure of $\mathbf{P}$ in $\Sigma$, then $\mathbf{P}^{*}$ is of degree of transcendency 1 over $\mathbf{K}$, and the pencil $\{W\}$ is composite with pencil $\left\{W^{*}\right\}$ defined by $\mathrm{P}^{*}$. Hence, a pencil $\{W\}$ is composite (with some other pencil) if and only if the field $\mathbf{P}$ which defines it is not maximally algebraic in $\Sigma$.

10. Theorem of Bertini (for pencils). If a pencil $\{W\}$, free from fixed components, is not composite, then all but a finite number of members of the pencil are irreducible varieties (over $\mathrm{K}$ ).

For the proof we first establish two very simple lemmas, probably well known.

Lemma 2. If $\mathrm{P}$ is a maximally algebraic subfield of a field $\Sigma$ and if $\Sigma^{*}=\Sigma\left(x_{1}, \cdots, x_{m}\right)$ is a pure transcendental extension of $\Sigma$, then $\mathrm{P}\left(x_{1}, \cdots, x_{m}\right)$ is maximally algebraic in $\Sigma^{*}$.

Proof. It is clearly sufficient to prove the lemma for $m=1$. Let then $\Sigma^{*}=\Sigma(x), x$ a transcendental over $\Sigma$, and let $t=f(x) / g(x)$ be an element of $\Sigma(x)$ which is algebraic over $\mathrm{P}(x)$. We assume that $f(x)$ and $g(x)$ are relatively prime (in $\Sigma[x]$ ). We have to prove that $t$ is an element of $\mathrm{P}(x)$. Consider first the case $t \in \Sigma$ (whence $f$ and $g$ are elements of $\Sigma$ ). Since $t$ is algebraic over $\mathrm{P}(x)$, it must be already algebraic over $\mathrm{P}$, since otherwise $x$ would be algebraic over $\mathrm{P}(t)$, and this is impossible, since $\mathrm{P}(t) \subset \Sigma$. Hence $t \in \mathrm{P}$, since $\mathrm{P}$ is maximally algebraic in $\Sigma$.

Let us now assume that $t$ is not in $\Sigma$. Then $t$, being a transcendental over $\Sigma$, is a fortiori transcendental over P. But $t$ is by hypothesis algebraic over $\mathrm{P}(x)$. Consequently $x$ is algebraic on: $\mathrm{P}(t)$. Let $f(x)=a_{0} x^{n}+\cdots+a_{n}$, $g(x)=b_{0} x^{n}+\cdots+b_{n} ; a_{0}, b_{0}$ not both zero, and $a_{i}, b_{j} \in \Sigma$. Then

$$
x^{n}\left(b_{0} t-a_{0}\right)+\cdots+\left(b_{n} t-a_{n}\right)=0,
$$

is the irreducible equation for $x$ over $\Sigma(t)$ (since $f(x)$ and $g(x)$ are relatively prime in $\Sigma[x])$. The $n$ roots of this equation are among the conjugates of $x$ over $\mathrm{P}(t)$, since $\mathrm{P}(t) \subset \Sigma(t)$. Consequently, the quotients

are algebraic over $\mathrm{P}(t)$.

$$
\zeta_{i}=\frac{b_{i} t-a_{i}}{b_{0} t-a_{0}}, \quad i=1,2, \cdots, n,
$$

Without loss of generality we may assume that $b_{0} \neq 0$ (we may replace $t$ by $1 / t)$. If we write down the algebraic equation for $\zeta_{i}$ over $\mathrm{P}(t)$, then after

constitute an $\mathfrak{S}_{\mathfrak{p}}$-module basis for $\mathfrak{S}_{\mathfrak{p}^{*}}^{*}$. Hence if $\omega \equiv c(\mathfrak{p})$ and if $\Delta, \Delta^{*}$ denote the residue fields at $\mathfrak{p}$ and $\mathfrak{p}^{*}$ respectively $\left(\Delta=\mathfrak{S} / \mathfrak{p}, \Delta^{*}=\mathfrak{S}^{*} / \mathfrak{p}^{*}\right)$, then $\Delta^{*}=\Delta(c)$, i.e., $\Delta^{*}=\Delta$, since $c \in K \subset \Delta$. This of course implies that $\Im^{*} \mathfrak{p} \neq \mathfrak{p}^{*}$, because $\Im^{*} \mathfrak{p}=\mathfrak{p}^{*}$ would imply the relation $\left[\Delta^{*}: \Delta\right]=\nu$. 
clearing the denominators we get an algebraic equation for $t$ over $\Sigma$. This must be an identity, since $t$ is transcendental over $\Sigma$. If we equate to zero the leading coefficient of this equation in $t$, we find that $b_{i} / b_{0}$ is algebraic over $\mathrm{P}$. But since $\mathbf{P}$ is maximally algebraic in $\Sigma$, it follows that $b_{i} / b_{0}$ is an element of $\mathbf{P}$. Hence we may write $g(x)=b_{0} \cdot g_{1}(x)$, where $g_{1}(x) \in \mathrm{P}[x]$.

If also $a_{0} \neq 0$, then replacing $t$ by $1 / t$ we conclude in the same fashion that $f(x)=a_{0} f_{1}(x), f_{1}(x) \in \mathrm{P}[x]$. We have now

$$
t=\frac{a_{0}}{b_{0}} \cdot \frac{f_{1}(x)}{g_{1}(x)} .
$$

Since $t$ is algebraic over $\mathrm{P}(x)$ and since $f_{1}(x) / g_{1}(x) \in \mathrm{P}(x)$, it follows that $a_{0} / b_{0}$ is algebraic over $\mathrm{P}(x)$. But since $a_{0} / b_{0} \in \Sigma$, it follows by the preceding case that $a_{0} / b_{0} \in \mathrm{P}$, whence $t \in \mathrm{P}(x)$. If $a_{0}=0$, then we replace $t$ by $c+t$ where $c \in \mathrm{P}$ and $c \neq 0$. Then $c+t$ is a quotient of two polynomials, $(c g+f) / g$, of the same degree. By the preceding case $a_{0} \neq 0$, it follows that $c+t \in \mathrm{P}(x)$, whence also $t \in \mathbf{P}(x)$. This completes the proof of the lemma.

Lemma 3. Let $f\left(x_{1}, \cdots, x_{n}\right)$ be an irreducible polynomial in $\mathrm{P}\left[x_{1}, \cdots, x_{n}\right]$, where $\mathrm{P}$ is a field, and let $\Sigma$ denote the field $\mathrm{P}\left(\xi_{1}, \cdots, \xi_{n}\right)$ defined by the equation $f\left(\xi_{1}, \cdots, \xi_{n}\right)=0$. If $\mathrm{P}$ is maximally algebraic in $\Sigma$, then the polynomial $f\left(x_{1}, \cdots, x_{n}\right)$ is absolutely irreducible.

Proof. It is sufficient to prove that $f$ remains irreducible for any finite extension $\Omega$ of P. The lemma is trivial when the field $\Omega$ is a pure transcendental extension of $\mathrm{P}: \Omega=\mathrm{P}\left(u_{1}, \cdots, u_{m}\right)$. Moreover, in this case, if $\Sigma^{*}$ denotes the field $\Omega\left(\xi_{1}, \cdots, \xi_{n}\right)$, then $\Sigma^{*}$ is a pure transcendental extension of $\Sigma$, namely: $\Sigma^{*}=\Sigma\left(u_{1}, \cdots, u_{m}\right)$. Since $\mathrm{P}$ is maximally algebraic in $\Sigma$, it follows, by the preceding lemma, that $\Omega$ is maximally algebraic in $\Sigma^{*}$. We conclude that it is sufficient to prove the lemma for finite algebraic extensions of $\mathrm{P}$. But in this case the len:ma follows from a more general result concerning the behavior of prime ideals under algebraic extensions of the ground field (Zariski [5, Theorem 3, p. 198]).

11. Proof of the theorem of Bertini. The notation being that of $\S 3$, let $\zeta$ be any element of $\Sigma^{*}$ and let

$$
F\left(\left\{u_{i j}\right\},\left\{\eta_{i}\right\}, \zeta\right)=0
$$

be the irreducible equation for $\zeta$ over the field $\mathrm{P}^{*}\left(=\mathrm{P}\left(\left\{u_{i j}\right\},\left\{\eta_{i}\right\}\right)\right)$. We may assume that $F$ is an irreducible polynomial in $\mathrm{P}\left[\left\{u_{i j}\right\},\left\{\eta_{i}\right\}, \zeta\right]$. Since $\Sigma^{*}$ is a pure transcendental extension of $\Sigma$ and since, by hypothesis, $\mathbf{P}$ is maximally algebraic in $\Sigma$, it follows, by Lemma 2 , that $\mathbf{P}$ is also maximally algebraic in $\Sigma^{*}$. Hence, a fortiori, $\mathrm{P}$ is maximally algebraic in the field $\mathrm{P}\left(\left\{u_{i j}\right\},\left\{\eta_{i}\right\}, \zeta\right)$, a subfield of $\Sigma^{*}$. By Lemma 3, we conclude that the polynomial $F$ is absolutely irreducible: 
Let $a, b, \cdots$ be the coefficients of the general polynomial in the arguments $\left\{u_{i j}\right\},\left\{\eta_{i}\right\}, \zeta$, of the same degree as $F(a, b, \cdots$ are indeterminates). Let $a_{0}, b_{0}, \cdots$ be the corresponding coefficients of $F\left(a_{0}, b_{0}, \cdots\right.$ are elements of $\mathrm{P}$ ). There exists a finite number of finite sets of polynomials in $a, b, \cdots$, with rational coefficients, say

$$
\left\{G_{i 1}(a, b, \cdots), G_{i 2}(a, b, \cdots), \cdots\right\}, \quad i=1,2, \cdots, \rho,
$$

with the following property, that if $F^{*}\left(\left\{u_{i j}\right\},\left\{\eta_{i}\right\}, \zeta\right)$ is a polynomial, of the same degree as $F$, with coefficients $\alpha, \beta, \ldots$ in some field $\Omega$, a necessary and sufficient condition that $F^{*}$ be reducible in some extension of $\Omega$ is that $G_{i 1}(\alpha, \beta, \cdots)=G_{i 2}(\alpha, \beta, \cdots)=\cdots=0$, for at least one value of $i$ $(i=1,2, \cdots, \rho)$. Since $F$ is absolutely irreducible, it follows that for each $i$ at least one of the elements

$$
G_{i 1}\left(a_{0}, b_{0}, \cdots\right), G_{i 2}\left(a_{0}, b_{0}, \cdots\right), \cdots
$$

is different from zero. These are elements of $P$. If then $\mathfrak{p}$ is a prime divisor of $\mathrm{P} / \mathrm{K}$, and if $a_{0}^{*}, b_{0}^{*}, \cdots$ denote the residues of $a_{0}, b_{0}, \cdots$ at $\mathfrak{p}$, then in the first place for all but a finite number of divisors $p$ the residues $a_{0}^{*}, b_{0}^{*}, \ldots$ will be all finite. In the second place, if an additional finite set of prime divisors $\mathfrak{p}$ is avoided, the expressions $G_{i 1}\left(a_{0}^{*}, b_{0}^{*}, \cdots\right), G_{i 2}\left(a_{0}^{*}, b_{0}^{*}, \cdots\right)$ will not be all zero, for any $i=1,2, \cdots, \rho$. Hence the resulting relation between the $B^{*}$ residues $u_{i}^{*}, \eta_{i}^{*}, \zeta^{*}$ :

$$
F^{*}\left(\left\{u_{i j}^{*}\right\},\left\{\eta_{i}^{*}\right\}, \zeta^{*}\right)=0
$$

remains absolutely irreducible, and in particular is irreducible over the residue field $\Delta$ of $\mathfrak{p}$. Here $B^{*}$ is the valuation of $\Sigma^{*}$ defined by one of the divisors $\mathfrak{B}_{i}^{*}$ in (2), say by $\Re_{1}^{*}$.

Let now $\zeta$ be a primitive element of $\Sigma^{*}$ over $\mathrm{P}^{*}$. Then $F$ is of degree $\nu$ in $\zeta$, where $\nu=\left[\Sigma^{*}: \mathrm{P}^{*}\right]$. Again, with the exception of a finite number of divisors $\mathfrak{p}$, the equation (9) will also be of degree $\nu$ in $\zeta^{*}$. Since this equation is irreducible, it follows that the relative degree of the residue field $\Delta^{*}$ of $\mathfrak{P}_{1}^{*}$ with respect to the field $\Delta\left(\left\{u_{i}^{*}\right\},\left\{\eta_{i}^{*}\right\}\right)$ is at least $\nu$. But this last field is the residue field of the prime divisor $\mathfrak{p}^{*}$ of $\mathrm{P}^{*} / \mathrm{K}^{*}$, and the relative degree in question cannot therefore exceed the relative degree $\left[\Sigma^{*}: \mathrm{P}^{*}\right]$. Hence

$$
\left[\Delta^{*}: \Delta\left(\left\{u_{i j}^{*}\right\},\left\{\eta_{i}^{*}\right\}\right)\right]=\nu .
$$

Now the relative degree $[\Delta: \mathrm{K}]$ is equal to $d$, where $d$ is the degree of $\mathfrak{p}$. Since the $u_{i j}^{*}, \eta_{i}^{*}$ are algebraically independent over $\Delta$, it follows that $\Delta\left(\left\{u^{*}\right\},\left\{\eta_{i}^{*}\right\}\right)$ is also of relative degree $d$ over the field $\mathrm{K}\left(\left\{u_{i j}^{*}\right\},\left\{\eta_{i}^{*}\right\}\right)$. Hence, by (10),

$$
\left[\Delta^{*}: \mathrm{K}\left(\left\{u_{i j}^{*}\right\},\left\{\eta_{i}^{*}\right\}\right)\right]=\nu d .
$$

But the left-hand side of this relation is the relative degree $d_{1}$ of $\mathfrak{B}_{1}^{*}$. Hence 
$d_{1}=\nu d$. Comparing with the relation (6) of $\S 7$, we conclude that $m=1, h_{1}=1$, whence $W_{\mathfrak{p}}=\Gamma_{1}$, and $W_{\mathfrak{p}}$ is an irreducible subvariety of $V$. Since this holds true for all but a finite number of prime divisors of $\mathrm{P} / \mathrm{K}$, the proof of the theorem of Bertini is complete.

12. Absolutely irreducible members of a non-composite pencil. In this section we wish to prove the following theorem:

If $\mathrm{K}$ is not maximally algebraic in $\Sigma$, then every irreducible subvariety of $V$ is absolutely reducible. If $\mathrm{K}$ is maximally algebraic in $\Sigma$, and if $\{W\}$ is a noncomposite pencil free from fixed components, then $a W_{\mathfrak{p}}$ is absolutely reducible if the degree of $\mathfrak{p}$ is $>1$, while, with a finite number of exceptions, all $W_{\mathfrak{p}}$ corresponding to prime divisors of degree 1 are absolutely irreducible.

For the proof of this theorem, we first recall the definition of absolute irreducibility. An irreducible variety $V$ in an $S_{n}\left(x_{1}, \cdots, x_{n}\right)$, over a ground field $\mathrm{K}$, is given by a prime ideal $\mathrm{p}$ in the polynomial ring $\Im=\mathrm{K}\left[x_{1}, \cdots, x_{n}\right]$. The variety $V$ is absolutely irreducible if $\mathfrak{p}$ remains prime under any extension $\mathrm{K}^{*}$ of the ground field $\mathrm{K}$; i.e., if $\mathfrak{\Im}^{*} \mathfrak{p}$ is prime, $\mathfrak{\Im}^{*}=\mathrm{K}^{*}\left[x_{1}, \cdots, x_{n}\right]$. In the contrary case, $V$ is absolutely reducible. We now derive a necessary and sufficient condition for absolute irreducibility:

Leмma 4. A necessary and sufficient condition in order that $V$ be absolutely irreducible is that $\mathrm{K}$ be maximally algebraic in the field $\Sigma$ of rational functions on $V\left({ }^{12}\right)$.

Proof. The condition is sufficient. If $\mathrm{K}^{*}$ is an algebraic extension of $\mathrm{K}$, then the sufficiency of the condition follows from the quoted theorem in $[5, \mathrm{p} .198]$. If $\mathrm{K}^{*}$ is a pure transcendental extension of $\mathrm{K}, \mathrm{K}^{*}=\mathrm{K}\left(u_{1}, \cdots, u_{m}\right), \Im^{*} \mathfrak{p}$ is prime unconditionally. Moreover, if $\Im^{*} \mathfrak{p}=\mathfrak{p}^{*}$, then the quotient field $\Sigma^{*}$ of $\mathfrak{S}^{*} / \mathfrak{p}^{*}$ coincides with the field $\Sigma\left(u_{1}, \cdots, u_{m}\right)$, a pure transcendental extension of $\Sigma$. By Lemma 2 , if $\mathrm{K}$ is maximally algebraic in $\Sigma, \mathrm{K}^{*}$ will be maximally algebraic in $\Sigma^{*}$. Hence $\mathfrak{p}^{*}$ remains prime under any algebraic extension of $\mathrm{K}^{*}$.

The condition is necessary. Let $\theta$ be an element of $\Sigma$ which is algebraic over $\mathrm{K}$, and let $F(\theta)=0$ be the irreducible equation for $\theta$ over $\mathrm{K}$. Let $\theta_{1}, \cdots, \theta_{0-1}$ be conjugates of $\theta$ over $\mathrm{K}$, where $g$ is the degree of $F$. We take as field $\mathrm{K}^{*}$ the field $\mathrm{K}\left(\theta, \theta_{1}, \cdots, \theta_{o-1}\right)$. Since $\theta \in \Sigma$, there must exist a quotient $\phi / \psi\left(\phi, \psi \in \mathrm{K}\left[x_{1}, \cdots, x_{n}\right]\right), \psi \neq \equiv 0(\mathfrak{p})$, such that $\psi^{\circ} F(\phi / \psi) \equiv 0(\mathfrak{p})$. This equation factors in $\Im^{*}:(\phi-\theta \psi)\left(\phi-\theta_{1} \psi\right) \cdots\left(\phi-\theta_{o-1} \psi\right) \equiv 0\left(\Im^{*} p\right)$. Now, by hypothesis, $\Im^{*} \mathfrak{p}$ is prime. Hence one of the factors $\phi-\theta_{i} \psi\left(\theta_{0}=\theta\right)$ must be in $\Im^{*} p$. Since $\Im^{*} p$ is invariant under all automorphisms of $K^{*}$ over $K$, it follows that all the $g$ factors $\phi-\theta_{i} \psi$ must be in $\Im^{*} p$. This implies that $g$ is 1 , since $\theta_{i} \neq \theta_{j}$ if $i \neq j$ and since $\psi \neq \equiv 0(\mathfrak{p})$. Hence $\theta \in \mathrm{K}$, and this shows that $\mathrm{K}$ is maximally algebraic in $\Sigma$.

${ }^{(2)}$ In Lemma 3 we have proved the sufficiency of this condition in the special case of principal ideals $(f)$. 
We now pass to the proof of the theorem stated at the beginning of this section.

Let us first assume that $\mathrm{K}$ is not maximally algebraic in $\Sigma$ (whence $V$ itself is absolutely reducible). Since $V$ is normal, the relative algebraic closure $\overline{\mathrm{K}}$ of $\mathrm{K}$ in $\Sigma$ must be contained in the integrally closed ring $\mathrm{D}=\mathrm{K}\left[\xi_{1}, \cdots, \xi_{n}\right]$. Hence $\overline{\mathrm{K}}$ is also contained in the quotient ring $\mathfrak{D} / \mathfrak{p}$ of any prime $\mathfrak{D}$-ideal $\mathfrak{p}$. Hence $\mathrm{K}$ is not maximally algebraic in the field of rational functions on the subvariety defined by $\mathfrak{p}$. Thus we conclude that in the present case $V$ does not carry at all absolutely irreducible subvarieties. Note that the hypothesis that $V$ is normal was essential in the proof.

We now consider the more interesting case in which the ground field $\mathrm{K}$ is maximally algebraic in $\Sigma$. Let $\mathfrak{p}$ be a prime divisor of $\mathrm{P} / \mathrm{K}$, of degree $d>1$. We show that not only $W_{\mathfrak{p}}$ but also any irreducible component $\Gamma$ of $W_{\mathfrak{p}}$ is absolutely reducible. Namely, the field $\Delta$ of rational functions on $\Gamma$ is the residue field of the prime divisor $\mathfrak{B}$ defined by $\Gamma$ (since $V$ is normal). Since $\mathfrak{B}$ induces in the field $\mathrm{P}$ the given prime divisor $\mathfrak{p}, \Delta$ contains the residue field of $\mathfrak{p}$. But since $d>1$, this last residue field is a proper algebraic extension of $K$. Hence $K$ is not maximally algebraic in $\Delta$, and $\Gamma$ is absolutely reducible.

There remains to consider the prime divisors $\mathfrak{p}$ of degree 1 . For these it is not difficult to see that the construction used in the proof of the theorem of Bertini actually leads to varieties $W_{\mathfrak{p}}$ which are not only irreducible over $\mathrm{K}$ but are also absolutely irreducible. In fact, in the first place we have the absolutely irreducible equation (9). The coefficients of this equation are elements of the residue field of $p$, i.e., in the present case, elements of $K$. The absolute irreducibility of (9) implies, by Lemma 4 , that $\mathrm{K}$ is maximally algebraic in the field $\mathrm{K}\left(\left\{u_{i j}^{*}\right\},\left\{\eta_{i}^{*}\right\}, \zeta^{*}\right)$. In the second place, we may assume (avoiding a finite set of prime divisors $\mathfrak{p}$ ) that the equation (9) is of degree $\nu$ in $\zeta$, where $\nu=\left[\Sigma^{*}: \mathrm{P}^{*}\right]$. Then the field $\mathrm{K}\left(\left\{u_{i j}^{*}\right\},\left\{\eta_{i}^{*}\right\}, \zeta^{*}\right)$ coincides with the residue field $\Delta^{*}$ of $\mathfrak{P}_{1}^{*}$ (since this residue field is at most of relative degree $\nu$ with respect to the residue field of $\mathfrak{p}^{*}$, and since this last residue field is $\left.\mathrm{K}\left(\left\{u_{i j}^{*}\right\},\left\{\eta_{i}^{*}\right\}\right)\right)$. We conclude that $\mathrm{K}$ is maximally algebraic in $\Delta^{*}$. Since the field $\Delta$ of rational functions on the irreducible $W_{\mathfrak{p}}$ is a subfield of $\Delta^{*}$, it follows that $\mathrm{K}$ is maximally algebraic in $\Delta$ and that consequently $W_{\mathfrak{p}}$ is absolutely irreducible (Lemma 4).

\section{LINEAR SYSTEMS OF ARBITRARY DIMENSION}

13. Definitions. $A$ pencil $\{W\}$ is linear if the corresponding field $\mathrm{P}$ is a simple transcendental extension of $\mathrm{K}$ (if $\{W\}$ has fixed components, $\mathrm{P}$ is the field which defines the pencil obtained from $\{W\}$ by deleting these fixed components).

Let us assume that $\{W\}$ is linear, and let $\mathrm{P}=\mathrm{K}(t), t$ a transcendental over $\mathrm{K}, t \in \Sigma$. From our definition of a pencil, given in $\$ 2$, it follows that our linear pencil $\{W\}$ can be obtained in the following fashion. Let $p$ be a prime 
divisor of $\mathrm{P} / \mathrm{K}$ and let us assume that $\mathfrak{p}$ is not the divisor at infinity of $(t)$. Let $f\left(t^{*}\right)=0$ be the irreducible equation over $\mathrm{K}$, of degree $d$, satisfied by the residue $t^{*}$ of $t$ at $\mathfrak{p}$. Here $d$ is the degree of $\mathfrak{p}$. The principal divisor $(f(t))$ in $\Sigma$ can be written in the form:

$$
(f(t))=\mathfrak{A}_{\mathfrak{p}} / \mathfrak{\Re}_{\infty}^{d},
$$

where $\mathfrak{A}_{\mathfrak{p}}, \mathfrak{A}_{\infty}$ are integral divisors (of the first kind) and $\mathfrak{A}_{\infty}$ is the denominator of the principal divisor $(t)$ and is independent of $\mathfrak{p}$. The member $W_{\mathfrak{p}}$ of the pencil $\{W\}$, outside of possible fixed components, is the subvariety of $V$ which corresponds to the integral divisor $\mathfrak{A}_{\mathfrak{p}}$. If $\mathfrak{p}$ is the prime divisor where $t$ becomes infinite, then $\mathfrak{A}_{\mathfrak{p}}=\mathfrak{A}_{\infty}$ and $d=1$.

Suppose now that we consider only those prime divisors of $\mathrm{P} / \mathrm{K}$ which are of degree $d=1$. Then $f(t)=c_{0} t+c_{1}, c_{0}, c_{1} \in \mathrm{K}$, and we will have

$$
\left(c_{0} t+c_{1}\right)=\frac{\mathfrak{A}_{\mathfrak{p}}}{\mathfrak{A}_{\infty}} \text {. }
$$

This suggests a possible definition of a linear pencil which is different from ours. Namely one could define $\{W\}$ as the set of all $W_{\mathfrak{p}}$ for which $\mathfrak{p}$ is of degree 1 . This is the customary definition in classical algebraic geometry. Unless $\mathrm{K}$ is algebraically closed, these usual pencils are proper subsets of our pencils. They are defined by the linear one-parameter family of functions: $c_{0} t+c_{1}\left({ }^{13}\right)$. We use this customary procedure as a basis for the definition of linear systems. This definition is well known. We consider namely a finite set of functions in $\Sigma$, say $t_{1}, \cdots, t_{s}$, such that $1, t_{1}, \cdots, t_{s}$ are linearly independent over $\mathrm{K}$. For arbitrary constants $c_{0}, c_{1}, \cdots, c_{s}$ in $\mathrm{K}$, not all zero, let

$$
c_{0}+c_{1} t_{1}+\cdots+c_{s} t_{s}=\mathfrak{A}_{(c)} / \mathfrak{A}_{\infty},
$$

where $\mathfrak{A}_{(c)}$ and $\mathfrak{A}_{\infty}$ are integral divisors (of the first kind) and where $\mathfrak{A}_{\infty}$ is independent of the $c$ 's. Let $W_{(c)}$ be the $(r-1)$-dimensional subvariety of $V$ which corresponds to $\mathfrak{A}_{(c)}$. The totality of all $W_{(c)}$, as the constants $c_{i}$ vary in $\mathbf{K}$, is called a linear system of dimensions $s$, and is denoted by $|W|$. It is clear that $\mathfrak{A}_{\infty}$ itself is a particular $\mathfrak{A}_{(c)}$, namely for $c_{0}=1, c_{1}=\cdots=c_{s}=0$. It is also clear that we can always assume that no prime factor of $\mathfrak{A}_{\infty}$ occurs in all $\mathfrak{A}_{(c)}$ and that by this condition $\mathfrak{A}_{\infty}$ is uniquely determined. The system $|W|$ is then free from fixed components. If $\mathfrak{A}_{\infty}$ is chosen in this fashion, and if $\mathfrak{R}$ is an arbitrary fixed integral divisor, then $\mathfrak{A}_{(c)} \cdot \mathfrak{l} / \mathfrak{A}_{\infty} \cdot \mathfrak{l}$ is another representation of the principal divisor $\left(c_{0}+c_{1} t+\cdots+c_{s} t\right)$, but it is not in reduced form. The system $|W|$ defined by this representation would have as fixed component the $(r-1)$-dimensional subvariety which corresponds to \&.

(13) All the results proved in the preceding sections continue to hold for these pencils, except the property that through each point of $V$ there passes at least one $W_{\mathfrak{p}}$. 
We note that if $|W|$ is free from fixed components and if

$$
\mathfrak{A}_{\infty}=\mathfrak{B}_{\infty 1}^{h_{1}} \cdots \mathfrak{P}_{\infty m}^{h_{m}},
$$

then each $t_{i}$ has at $\mathfrak{B}_{\infty j}$ order $\geqq-h_{j}$, and at least one function $t_{i}$ must have at $\mathfrak{B}_{\infty j}$ order exactly equal to $-h_{j}$.

One more remark. If we pass from $t_{1}, \cdots, t_{s}$ to another set of functions $\tau_{1}, \cdots, \tau_{s}$, by a nonsingular transformation of the form

$$
\tau_{i}=\frac{d_{i 0}+d_{i 1} t_{1}+\cdots+d_{i s} t_{s}}{d_{00}+d_{01} t_{1}+\cdots+d_{0 s} t_{s}}
$$

then the functions $1, \tau_{1}, \cdots, \tau_{s}$ define the same linear system $|W|$. Namely, we will have

$$
\left(b_{0}+b_{1} \tau_{1}+\cdots+b_{s} \tau_{s}\right)=\frac{\mathfrak{A}_{\left(c^{\prime}\right)}}{\mathfrak{U}_{\left(d_{0 j}\right)}},
$$

where $c_{j}^{\prime}=b_{0} d_{0 j}+b_{1} d_{1 j}+\cdots+b_{s} d_{s j}$.

14. Composite linear systems. Let $\Omega=\mathrm{K}\left(t_{1}, \cdots, t_{s}\right)$ and let us assume that $\Omega$ is of degree of transcendency 1 over $\mathrm{K}$. Let us see what geometric property of $|W|$ corresponds to this assumption. We shall also assume that $|W|$ is free from fixed components.

Let $\{Z\}$ be the pencil (free from fixed components) defined by the field $\Omega$. Let $t=c_{0}+c_{1} t_{1}+\cdots+c_{s} t_{s}$, where $c_{0}, \cdots, c_{s}$ are arbitrary but fixed constants. The field $\mathrm{K}(t)=\mathbf{P}$ defines a pencil $\left\{W^{\prime}\right\}$, free from fixed components. It is clear that each $W^{\prime}$ is either a $W$, or becomes a $W$ after a certain fixed component $M_{0}$ is added to each $W^{\prime}$. Namely, if $t$ has order $-q_{i}$ at $\mathfrak{P}_{\infty i}$ $(i=1,2, \cdots, m)$, and if $\Gamma_{\infty i}$ is the irreducible $V_{r-1}$ which corresponds to $\mathfrak{P}_{\infty i}$, then $W^{\prime}+\left(h_{1}-q_{1}\right) \Gamma_{\infty 1}+\cdots+\left(h_{m}-q_{m}\right) \Gamma_{\infty m}$ is a $W$, for each member $W^{\prime}$ of the pencil $\left\{W^{\prime}\right\}$.

Let the pencil $\left\{W^{\prime}+M_{0}\right\}$ be denoted by $\{W\}$. We have that $\mathrm{P}$ either coincides with $\Omega$ or is a subfield of $\Omega$. Let us first assume that $\{W\}$ has no fixed components. We assert that in this case $\mathrm{P}$ is a proper subfield of $\Omega$, provided $s>1$.

To show this, let us assume the contrary. Each $t_{i}$ is then an element of $\mathrm{K}(t)$, say $t_{i}=f_{i}(t) / g_{i}(t)$, where $f_{i}$ and $g_{i}$ are assumed to be relatively prime. Let $t-d$ be a factor of $g_{i}(t)$, if $g_{i}$ is of degree $\geqq 1$. Let $\mathfrak{B}$ be a prime divisor of $\Sigma / \mathrm{K}$ which occurs in the numerator of the principal divisor $(t-d)$. Since $\{W\}$ has no fixed components, $t$, and hence also $t-d$, becomes infinite along each of the varieties $\Gamma_{\infty 1}, \cdots, \Gamma_{\infty m}$. Consequently $\mathfrak{B} \neq \mathfrak{B}_{\infty i}, i=1,2, \cdots, m$. On the other hand, since $t-d$ is a factor of $g_{i}(t), t_{i}$ is infinite at the divisor $\mathfrak{B}$, and this is impossible since $t_{i}$ can become infinite only at $\mathfrak{P}_{\infty 1}, \cdots, \mathfrak{B}_{\infty m}$.

Hence $g_{i}(t)$ is of degree zero, i.e., $t_{i}=f_{i}(t)$. Let $\nu_{i}$ be the degree of $f_{i}(t)$. Again, since $\{W\}$ is free from fixed components, the order of $t$ at $\mathfrak{B}_{\infty}$ is ex- 
actly $-h_{j}$. Hence the order of $t_{i}$ at $\mathfrak{B}_{\infty j}$ is exactly $-h_{j} \nu$. But this order is at most $-h_{j}$. Hence $\nu \leqq 1$. Thus each $t_{i}$ is of the form: $t_{i}=d_{i 0}+d_{i 1} t$, and if $s>1$ this is impossible, since $1, t_{1}, \cdots, t_{s}$ are linearly independent over $\mathrm{K}$. This proves our assertion.

Since $\{W\}$ has no fixed components, every $W^{\prime}$ is a $W$, and since $\Omega$ is a proper algebraic extension of $P,\{W\}$ is composite with the pencil $\{Z\}$. We have therefore proved that each $W$ decomposes in to a certain number of varieties $Z$, provided $W$ belongs to some subpencil of $|W|$ which is free from fixed components. But it is clear that there exists such a pencil for each $W$. Namely, let $W=W_{(c)}$ and let $W_{\left(c^{\prime}\right)}$ be a $W$ which has no components in common with $W_{(c)}$. We put

$$
t=\frac{c_{0}+c_{1} t_{1}+\cdots+c_{s} t_{s}}{c_{0}^{\prime}+c_{1}^{\prime} t_{1}+\cdots+c_{s}^{\prime} t_{s}} .
$$

It is then clear that the pencil determined by the field $\mathrm{K}(t)$ is contained in the linear system $|W|$, is free from fixed components, and that $W_{(c)}$ and $W_{\left(c^{\prime}\right)}$ are members of that pencil.

In conclusion, we have proved that if $s>1$ and if $\mathrm{K}\left(t_{1}, \cdots, t_{s}\right)$ is of degree of transcendency 1 over $\mathrm{K}$, then each member of the linear system $|W|$ (free from fixed components) decomposes into a certain number of varieties $Z$. We say that $|W|$ is composite with the pencil $\{Z\}$.

15. The theorem of Bertini for linear systems. We shall say that a linear system $|W|$ is reducible if each member $W$ of the system is reducible over $\mathrm{K}$. We shall say that $|W|$ is absolutely reducible, if each $W$ is absolutely reducible.

Theorem of Bertini. A reducible linear system $|W|$, free from fixed components, is necessarily composite with a pencil. If $\mathrm{K}$ is maximally algebraic in $\Sigma$, then the assumption that $|W|$ is reducible can be replaced, by the weaker assumption that $|W|$ is absolutely reducible.

For linear systems of dimension 1 this theorem has already been proved in $\S \S 11$ and 12 . In order to prove this theorem for system of dimension $s>1$, we first prove a lemma.

Lemma 5. Let $\Sigma$ be a field of algebraic functions of $r$ independent variables, $r>1$, and let the ground field $\mathrm{K}$ be maximally algebraic in $\Sigma$. If $x_{1}, \cdots, x_{\rho}$, $1<\rho \leqq$ r are algebraically independent elements of $\Sigma$ (over $\mathrm{K}$ ) and if we put

$$
\bar{x}_{i}=c_{i 1} x_{1}+\cdots+c_{i \rho} x_{\rho}, \quad i=1,2, \cdots, \rho-1,
$$

then for non-special constants $c_{i j}$ in $\mathrm{K}$, the field $\mathrm{K}\left(\bar{x}_{1}, \cdots, \bar{x}_{\rho-1}\right)$ is maximally algebraic in $\Sigma$.

Proof. If the lemma is true for $\rho=2$, then for non-special constants $c_{1}, c_{2}$ the field $\overline{\mathrm{K}}=\mathrm{K}\left(c_{1} x_{1}+c_{2} x_{2}\right)$ is a maximally algebraic subfield of $\Sigma$, and the de- 
gree of transcendency of $\Sigma$ over $\overline{\mathrm{K}}$ is $r-1$. If, say, $c_{1} \neq 0$, then $x_{2}, \cdots, x_{\rho}$ are algebraically independent over $\overline{\mathrm{K}}$, and hence replacing $\mathrm{K}$ by $\overline{\mathrm{K}}$ we achieve a reduction from $\rho$ to $\rho-1$. Therefore it is sufficient to prove the lemma for $\rho=2$.

Let $\Sigma^{\prime}$ be the relative algebraic closure of the field $\mathrm{K}\left(x_{1}, x_{2}\right)$ in $\Sigma$. We may conduct the proof under the assumption that $\Sigma^{\prime}=\Sigma$, for if $\mathbf{K}\left(c_{1} x_{1}+c_{2} x_{2}\right)$ is maximally algebraic in $\Sigma^{\prime}$, then it is also maximally algebraic in $\Sigma$. Hence we may assume that $r=2$ and that therefore $\Sigma$ is an algebraic extension of $\mathrm{K}\left(x_{1}, x_{2}\right)$. Since we are dealing with fields of algebraic functions, $\Sigma$ is a finite extension of $\mathrm{K}\left(x_{1}, x_{2}\right)$.

Let $\bar{x}=x_{1}+c x_{2}, c \in \mathrm{K}$, and let $\Omega_{c}$ denote the relative algebraic closure of the field $\mathrm{K}(\bar{x})$ in $\Sigma$. We shall prove that for all but a finite number of elements $c$ in $\mathrm{K}$ the field $\Omega_{c}$ coincides with $\mathrm{K}(\bar{x})$. This result will establish the lemma.

Let $\Sigma_{c}=\Omega_{c}\left(x_{2}\right)$. We have

$$
\mathrm{K}\left(x_{1}, x_{2}\right) \subseteq \Sigma_{c} \subseteq \Sigma
$$

Since $\Sigma$ is a finite algebraic extension of $\mathrm{K}\left(x_{1}, x_{2}\right)$ and since for each $c$ in $\mathrm{K}$ the field $\Sigma_{c}$ is between $\mathrm{K}\left(x_{1}, x_{2}\right)$ and $\Sigma$, it follows that there is only a finite number of distinct fields $\Sigma_{c}\left({ }^{14}\right)$. Therefore, for all but a finite number of elements $c$ in $\mathbf{K}$, it is true that for a given $c$ in $\mathrm{K}$ there exists another element $d$ in $\mathrm{K}, d \neq c$, such that $\Sigma_{c}=\Sigma_{d}$. We proceed to prove that for any such element $c$ in $\mathrm{K}$, the field $\Omega_{c}$ coincides with $\mathrm{K}(\bar{x})$.

We may identify $x_{1}+c x_{2}$ and $x_{1}+d x_{2}$ with $x_{1}$ and $x_{2}$ respectively, since $c \neq d$. The fields $\Omega_{c}$ and $\Omega_{d}$ are now the relative algebraic closures in $\Sigma$ of the fields $\mathrm{K}\left(x_{1}\right)$ and $\mathrm{K}\left(x_{2}\right)$ respectively. They shall be denoted by $\Omega_{1}$ and $\Omega_{2}$ respectively. Similarly we put $\Sigma_{1}=\Omega_{1}\left(x_{2}\right), \Sigma_{2}=\Omega_{2}\left(x_{1}\right)$. We have, by hypothesis, $\Sigma_{1}=\Sigma_{2}=\Sigma^{*}$. The field $\mathrm{K}$ was assumed to be maximally algebraic in $\Sigma$. Hence $\mathrm{K}$ is also maximally algebraic in $\Omega_{2}$. Since $\Sigma^{*}\left(=\Sigma_{2}\right)$ is a pure transcendental extension of $\Omega_{2}$, it follows, by Lemma 2 of $\$ 10$, that $\mathrm{K}\left(x_{1}\right)$ is maximally algebraic in $\Sigma^{*}$. But $\Sigma^{*}=\Sigma_{1} \supset \Omega_{1}$ and $\Omega_{1}$ is algebraic over $\mathrm{K}\left(x_{1}\right)$. Hence $\Omega_{1}=\mathrm{K}\left(x_{1}\right)$, as was asserted.

16. Proof of the theorem of Bertini. By a linear homogeneous transformation on $t_{1}, \cdots, t_{s}$ we may arrange matters so that $t_{1}$ becomes infinite on $\Gamma_{\infty i}$ $(i=1,2, \cdots, m)$ to the highest order $h_{i}$. We consider the pencil $\{W\}$ contained in the system $|W|$ and defined by the functions $1, t_{1}+c t_{i}$, where $c \in \mathrm{K}$. For all but a finite number of constants $c$ in $\mathrm{K}, t_{1}+c t_{i}$ becomes infinite on $\Gamma_{\infty i}$ to the order $h_{i}$. Avoiding the exceptional constants $c$, we may therefore assert that the pencil $\{W\}$ is free from fixed components and consequently coincides with the pencil determined by the field $\mathrm{K}\left(t_{1}+c t_{i}\right)$. However, the pencil $\{W\}$ contains only those varieties $W_{\mathfrak{p}}$ which correspond to prime divisors $\mathfrak{p}$ of

(14) Note that $\mathbf{K}$ is of characteristic zero and hence that $\Sigma$ is a separable extension of $\mathrm{K}\left(x_{1}, x_{2}\right)$. If we did not assume that $\mathrm{K}$ is of characteristic zero we could not assert that there is only a finite number of fields between $\mathrm{K}\left(x_{1}, x_{2}\right)$ and $\Sigma$. 
$\mathbf{K}\left(t_{1}+c t_{i}\right)$ which are of degree 1 . Let $\mathbf{K}^{*}$ be the relative algebraic closure of $\mathbf{K}$ in $\Sigma$, and let $\left\{W^{*}\right\}$ be the pencil defined by the field $\mathrm{K}^{*}\left(t_{1}+c t_{i}\right)$. Again we include in $\left\{W^{*}\right\}$ only those varieties $W_{\mathfrak{p}^{*}}^{*}$ which correspond to prime divisors $\mathfrak{p}^{*}$ of $\mathrm{K}^{*}\left(t_{1}+c t_{i}\right)$ which are of degree 1 . If we identify the fields $\mathrm{K}\left(t_{1}+c t_{i}\right), \mathrm{K}^{*}\left(t_{1}+c t_{i}\right)$ with the fields $\mathbf{P}, \mathbf{P}^{*}$ respectively, of $\S 9$, we notice that if $\mathfrak{p}$ is of degree 1 , then $\mathfrak{p}$ extends to a unique prime divisor of $\mathfrak{p}^{*}$ of $\mathrm{P}^{*}$, i.e., we will have in (7): $s=1, \rho_{1}=1$. Hence each member of the pencil $\{W\}$ is also a member of the pencil $\left\{W^{*}\right\}$. By hypothesis, each $W$ is reducible over $\mathrm{K}$. Hence, by the theorem of Bertini for pencils, the field $\mathrm{K}^{*}\left(t_{1}+c t_{i}\right)$ is not maximally algebraic in $\Sigma$. The same conclusion holds true if $\mathbf{K}^{*}=\mathbf{K}$ and if each $W$ is absolutely reducible, in view of the theorem of $\$ 12$. Since this is true for all but a finite number of constants $c$ in $\mathrm{K}$, it follows, by Lemma 5 , that $t_{1}$ and $t_{i}$ are algebraically dependent over $\mathrm{K}^{*}$, and hence also over $\mathrm{K}$. Since this holds for $i=2, \cdots, s$ and since $t_{1}$ is a transcendental over $\mathrm{K}$, it follows that the field $\mathrm{K}\left(t_{1}, \cdots, t_{s}\right)$ is an algebraic extension of $K\left(t_{1}\right)$ and is therefore of degree of transcendency 1 , q.e.d.

\section{REFERENCES}

1. W. Krull, Idealtheorie, Ergebnisse der Mathematik und ihrer Grenzgebiete, vol. 4, no. 3.

2. B. L. van der Waerden, Moderne Algebra, vol. 2.

3. B. L. van der Waerden, Über lineare Scharen von reduziblen Mannigfaltigkeiten, Mathematische Annalen, vol. 113 (1936).

4. O. Zariski, Some results in the arithmetic theory of algebraic varieties, American Journal of Mathematics, vol. 61 (1939).

5. O. Zariski, Algebraic varieties over ground fields of characteristic zero, American Journal of Mathematics, vol. 62 (1940).

The Johns Hopkins University, BALTIMORE, MD.

California Institute of Technology, Pasadena, Calif. 